\title{
iRGD Peptide-Mediated Liposomal Nanoparticles with Photoacoustic/Ultrasound Dual-Modality Imaging for Precision Theranostics Against Hepatocellular Carcinoma
}

\author{
Huipu $\mathrm{Li}^{1, *}$ \\ Shasha $\mathrm{Shi}^{1}{ }^{1} *$ \\ Meng $\mathrm{Wu}^{2, *}$ \\ Wei Shen' \\ Jianli $\operatorname{Ren}^{3}$ \\ Zhechuan Mei (D) \\ Haitao $\operatorname{Ran}^{3}$ \\ Zhigang Wang $\mathbb{D}^{3}$ \\ Yi Tian ${ }^{4}$ \\ Jian Gao \\ Hongyun Zhao ${ }^{1,3}$ \\ 'Department of Gastroenterology, The \\ Second Affiliated Hospital of Chongqing \\ Medical University, Chongqing, People's \\ Republic of China; ${ }^{2}$ Department of \\ Ultrasound, Zhongnan Hospital of \\ Wuhan University, Wuhan, Hubei, \\ People's Republic of China; ${ }^{3}$ Chongqing \\ Key Laboratory of Ultrasound Molecular \\ Imaging, The Second Affiliated Hospital of \\ Chongqing Medical University, \\ Chongqing, People's Republic of China; \\ ${ }^{4}$ Department of Plastic Surgery, The \\ Second Affiliated Hospital of Chongqing \\ Medical University, Chongqing, People's \\ Republic of China \\ *These authors contributed equally to \\ this work
}

Correspondence: Hongyun Zhao; Jian Gao Email zhychina@cqmu.edu.cn; 982213482@qq.com
Purpose: Prepare a multifunctional ultrasound molecular probe, cell-penetrating peptidemodified 10-hydroxycamptothecin-loaded phase-transformation lipid nanoparticles (iRGDICG-10-HCPT-PFP-NPs), and to combine iRGD-ICG-10-HCPT-PFP -NPs with low-intensity focused ultrasound (LIFU) for precision theranostics against hepatocellular carcinoma (HCC). Materials and Methods: The morphology of nanoparticles (NPs) and iRGD-ICG-10HCPT-PFP-NPs was detected. In vitro, we examined targeting ability by flow cytometry and confocal laser scanning microscopy (CLSM), assessed penetration ability into hepatoma cells, and assessed killing ability. In vivo, we examined the targeting ability of the NPs with a photoacoustic (PA) imager and fluorometer (FL), while LIFU irradiation was used to trigger the release of chemotherapeutic drugs, which had a therapeutic effect on tumors.

Results: The particle size of iRGD-ICG-10-HCPT-PFP-NPs was $298.4 \pm 10.42 \mathrm{~nm}$. In vitro, iRGD-ICG-10-HCPT-PFP-NPs bound more to SK-Hep1 cells than ICG-10-HCPT-PFP-NPs. iRGD-ICG-10-HCPT-PFP-NPs could achieve PA/ultrasound imaging. The percentage of antiproliferative and apoptotic cells in the iRGD-ICG-10-HCPT-PFP-NPs+LIFU group was significantly higher. In vivo, iRGD-ICG-10-HCPT-PFP-NPs can target tumor sites and achieve PA/ ultrasound imaging. The tumor volume in the iRGD-ICG-10-HCPT-PFP-NPs+LIFU group was significantly smaller, and the antiproliferative and proapoptotic effects were higher.

Conclusion: We successfully prepared a novel molecular probe that has good targeting, can perform ultrasound/PA dual-modality imaging, and can penetrate deep into tumors to achieve better therapeutic tumor effects, providing a new idea and method for theranostics of HCC. Keywords: molecular probe, homing membrane peptide, precision diagnosis and treatment, dual-modality imaging

\section{Introduction}

Hepatocellular carcinoma (HCC), a malignant tumor that has long-afflicted and threatened human life and health, has a high incidence and mortality, and early diagnosis of this disease is difficult. Once diagnosed in the late stage, HCC brings heavy economic and life burdens to patients. ${ }^{1-3}$ The traditional treatment methods include surgical resection, transcatheter arterial embolization, and microwave ablation, which have the drawbacks of incomplete resection and a high recurrence rate; similarly, the side effects of systemic chemotherapy are great, and the lack of high sensitivity chemotherapeutic drugs also brings substantial difficulty to the treatment 
of HCC. ${ }^{4}$ Therefore, new ideas and directions need to be sought for the diagnosis and treatment of HCC.

At present, as an emerging diagnostic method, ultrasonic molecular imaging can be performed at the molecular level. ${ }^{5}$ Compared with the traditional imaging method, this approach uses a molecular probe with a smaller particle size as the medium for enhanced imaging, which can improve the diagnostic efficacy of imaging, detect the tumor lesion at an earlier stage, and diagnose the disease more accurately. ${ }^{6}$ At present, the molecular probe that has been put into clinical use is ultrasonic microbubbles, which can be used as an enhanced contrast agent for common B-mode ultrasound (US) and has certain clinical application value. $^{7}$ However, ultrasound microbubbles can only be imaged in blood vessels because of their large particle size, short circulation time, and inability to penetrate the tumor space, which is difficult to enrich at the tumor site to play a more accurate diagnostic role. ${ }^{8}$ Liposomal nanoparticles (NPs) are an emerging molecular probe that has been widely studied in recent years. ${ }^{9}$ They contain phospholipid bilayers and have good biocompatibility. At the same time, after polyethylene glycol modification, NPs are not easily phagocytosed by the reticuloendothelial system in vivo. ${ }^{10}$ With the help of the enhanced permeability and retention effect (EPR effect), they can reach the tumor site and accumulate to exert their effect. ${ }^{8,11,12}$ However, their numbers are obviously too low to rely solely on the EPR effect. ${ }^{13}$

Tumor homing peptides (THPs) with cell penetrating properties are a new type of small molecule peptide studied in recent years that can actively bind cell surface receptors and penetrate the intercellular space or even the cell membrane to deliver substances into the cell. ${ }^{14,15}$ Some peptides have the same sequence at the $\mathrm{C}$-terminus, and peptides expressing this sequence are able to promote phagocytosis of substances into the cell interior. ${ }^{16,17}$ Peptides containing this sequence can specifically bind to the neuromucin-1 (NRP-1) receptor, with a molecular weight of $103 \mathrm{kDa}$ and have the function of tissue penetration, a phenomenon known as the C-terminal rule (CendR) ${ }^{18,19}$ Only peptides with exposed arginine and lysine (exposed Cend) at the C-terminus can bind to NRP-1 and then enter endothelial cells. When there is a hidden $\mathrm{R}$ sequence inside the peptide chain, this sequence can be exposed, and this endocytosis process can be initiated by the hydrolysis of proteases. Many previous studies have confirmed that iRGD is a 9-amino acid cyclic peptide, and its sequence is that CRGDKGPDC actively targets tumor cells and promotes membrane penetration. ${ }^{16,20}$ Therefore, combining the iRGD peptide with liposomal NPs allows molecular probes to actively target tumor cells but also promotes NPs to penetrate the tumor cell membrane and deeply enter the interior of tumor cells.

Photoacoustic (PA) imaging is a new noninvasive and nonradioactive imaging method that has emerged in recent years. ${ }^{21-23}$ Its principle is that when the laser in the probe shines into the biological tissue, the substance containing light absorbers is injected into the biological tissue, and the light absorption domain of this substance will produce the ultrasonic signal. We call this ultrasonic signal generated by light excitation the PA signal. ${ }^{24}$ The PA signal generated in biological tissue carries the characteristic information of light absorption of the tissue, and the light absorption distribution image in the tissue can be reconstructed by detecting the PA signal. PA imaging combines the advantages of high selection characteristics in pure optical tissue imaging and deep penetration characteristics in pure ultrasonic tissue imaging, which can obtain highresolution and high-contrast tissue images, avoid the effect of light scattering from the principle, and realize deep in vivo tissue imaging of $50 \mathrm{~mm} .^{21,25}$ This imaging technology has the characteristics of real-time visibility and high efficiency. Indocyanine green (ICG), as the only light absorber approved by the US Food \& Drug Administration that can be used in the human body, has strong light absorption ability in the near-infrared range, is completely metabolized in vivo without toxic side effects and is a better choice for the development of clinically available PA molecular probes, so ICG encapsulation into liposomal NPs is used as a light absorber for PA imaging. ${ }^{26,27}$

As a systemic chemotherapeutic agent, 10-hydroxyc amptothecin (10-HCPT) has a short half-life, is lethal to tumor cells and is clinically applied in advanced chemotherapy of various cancers. ${ }^{28}$ However, as a systemic chemotherapeutic drug, it cannot avoid the common defects of drugs lacking targeting and is toxic to normal tissues and organs of the human body, especially in patients with advanced HCC in a cachectic state. The drug is therefore encapsulated into liposomal NPs to deliver the drug to the tumor site via actively targeted NPs, thereby achieving precise drug release. ${ }^{29,30}$ At the same time, the addition of liquid fluorocarbon to NPs, ${ }^{31}$ and it indicated that phase-transformation lipid NPs coated with liquid perfluoropentane (PFP) can elicit liquid-gas phase transition. Using a certain intensity of external ultrasonic irradiation, PFPcoated lipid NPs can be changed into lipid microbubbles to enhance ultrasound imaging. And the NPs were irradiated by low-intensity focused ultrasound (LIFU) developed by our laboratory, enables liquid fluorocarbon to undergo liquid-gas 
phase transition, which triggers nanoparticle rupture and promotes drug release, achieving a better therapeutic effect. ${ }^{32}$

Overall, as shown in Scheme 1, we intend to prepare a novel multifunctional dual-modality molecular probe (iRGD-ICG-10-HCPT-PFP-NPs) in this study equipped with iRGD peptides with active targeting and promoting tumor cell penetration, ICG with PA imaging ability, liquid fluorocarbon that produces liquid gas phase transition after LIFU irradiation, and the chemotherapeutic drug 10-HCPT. This method allows the nanoparticle to have the ability of ultrasonic/PA dual-modality imaging while the nanoparticle can actively target and penetrate the tumor cell membrane and release the drug after LIFU irradiation to achieve accurate killing of the tumor. For this reason, we performed the following experiments to examine the NP performance.

\section{Experimental}

\section{Materials and Cells}

Dipalmitoyl-phosphatidylcholine (DPPC) and cholesterol (Chol) were purchased from Avanti (USA). The iRGD peptide (sequence: CRGDKGPDC) was synthesized by ChinaPeptides (Shanghai, China). The DSPE-PEG2000Mal was obtained from Xian Ruixi Biotechnology (Xian, China). The DSPE-PEG2000-iRGD was synthesized by Funuowei Biotechnology (Chongqing, China). The 10HCPT (purity:99.9\%) was purchased from Lanbei Technology (Chengdu, China). The ICG was purchased from Sichuan Ikeda Technology (China). The Dioctadecyl3,3,3',3'-tetramethylindocarbocyanine (DiI) was provided by Beyotime Biotechnology (Shanghai, China). Calcein greenAM/propidium iodide PI and diamidino-phenylindole (DAPI) were obtained from Sigma-Aldrich (Saint Louis, USA). Dulbecco's modified Eagle's medium (DMEM) and fetal bovine serum (FBS) were purchased from Gibco. (Carlsbad, USA). A Cell Counting Kit 8 (CCK-8) was provided by Dojindo Technology (Tokyo, Japan).

Asonicator was purchased from Sonic (VCX-130; Sonics \& Materials, USA) along with a Yarong rotary vacuum evaporator (Shanghai, China). A confocal laser scanning microscope was obtained from Nikon (CLSM)(A1R-Si;

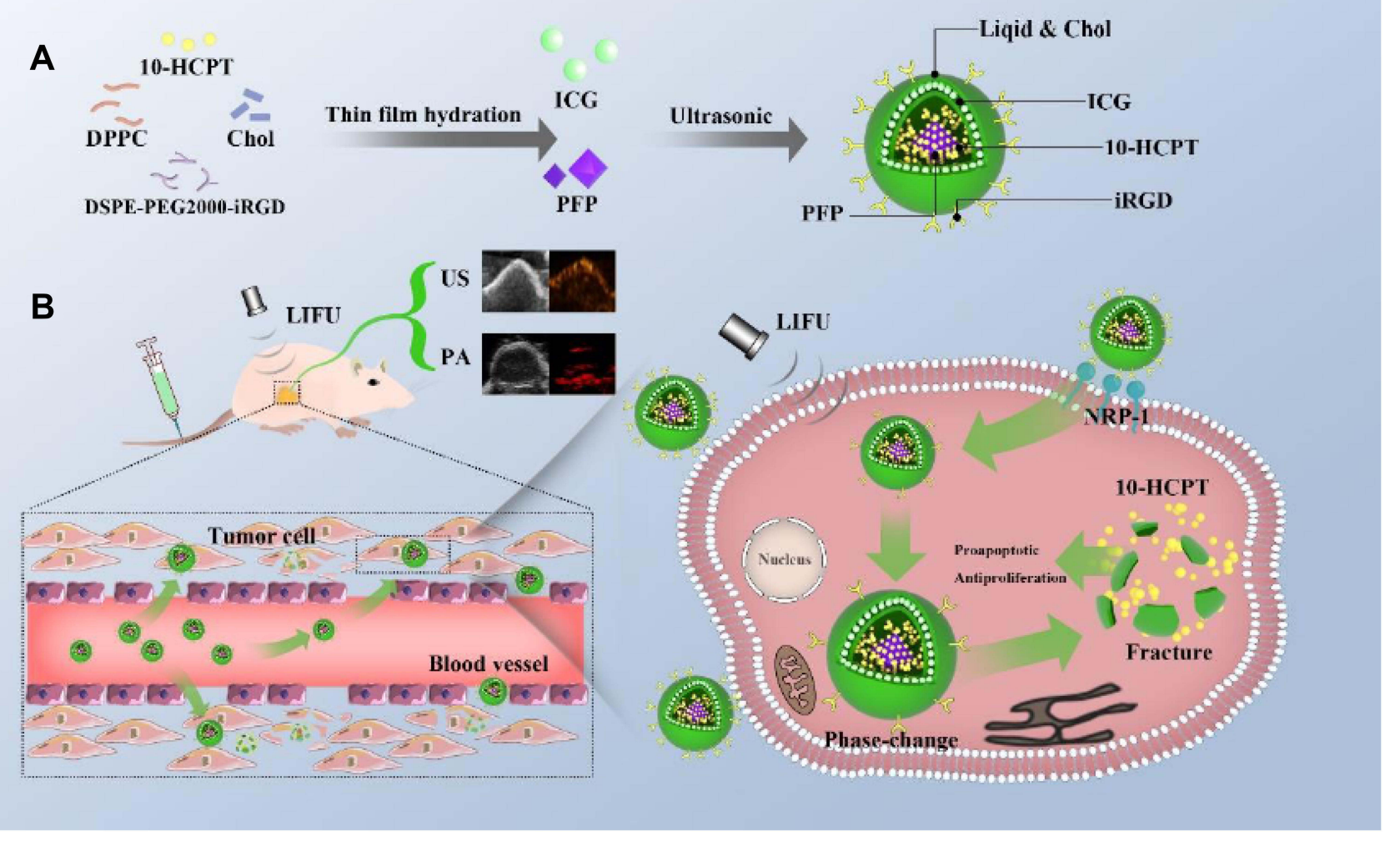

Scheme I (A) Preparation process of iRGD-ICG-I0-HCPT-PFP-NPs. (B) These nanoparticles are intelligent multifunctional theranostic agents for US/PA diagnostic imaging and targeted tumor therapy. The iRGD peptide is able to bind to tumor cells that highly express the NRP-I receptor, penetrate tumor cells and penetrate deeper into the interior of tumor cells, allowing iRGD peptide-mediated nanoparticles to enter tumor cells at a deeper level. After LIFU irradiation at the tumor site, the liquid PFP core in the nanoparticles undergoes a phase transition from liquid to gas. After the phase change, the NPs increase and eventually rupture, and when the NPs rupture, they produce a physical burst effect, release 10-HCPT chemotherapeutic drugs, reach the tumor interior, and produce a synergistic effect to achieve more accurate and effective treatment. 
Nikon, Tokyo, Japan) and a transmission electron microscope (TEM) (H-7500;Hitachi, Tokyo, Japan). A microplate reader (ELX800; Bio-tek Instruments,USA), dynamic light scattering analyzer (Malvern Instruments, UK) and LIFU instrument (LMSC051ACA; Institute of Ultrasound Imaging, Chongqing Medical Sciences, Chongqing, China) were used. A Vevo LAZR small animal photoacoustic imager was obtained from VisualSonics (VisualSonics, Canada).

A human HCC line (SK-Hep1) was purchased from Kunming Institute of Zoology, CAS (Kunming, China).

\section{NP Synthesis Method}

NPs were synthesized following the previously reported thin film hydration method and phacoemulsification method. $^{30,33,34}$ First, DPPC 5mg, DSPE-peg2000-iRGD $2 \mathrm{mg}$, cholesterol $1 \mathrm{mg}$, and 10-HCPT $1 \mathrm{mg}$ drug substance powder were added to a round-bottom flask and dissolved in $5 \mathrm{~mL}$ trichloromethane. The round-bottom flask was connected to a rotary evaporator and evaporated for 1 $\mathrm{h}$ at $52{ }^{\circ} \mathrm{C}$. After dissolving $1 \mathrm{mg}$ ICG in water, $200 \mu \mathrm{L}$ PFP was added, which was first emulsified with an ultrasonic sonicator at $100 \mathrm{~W}$ ( $5 \mathrm{~s}$ on, $5 \mathrm{~s}$ off) for $3 \mathrm{~min}$, and then all products were emulsified at the same power for 8 min. Finally, iRGD-ICG-10-HCPT-PFP-NPs were prepared by centrifugation at $8000 \mathrm{rpm}$ for $5 \mathrm{~min}$ at $4{ }^{\circ} \mathrm{C}$ in a centrifuge, discarding the supernatant, and resuspending with $2 \mathrm{~mL}$ PBS solution three times. ICG-10-HCPT-PFPNPs were obtained by the same procedures described above, except that DSPE was used instead of DSPE-iRGD.

\section{NP Characterization Testing}

The prepared NPs were diluted at a concentration of $500 \mathrm{mg} / \mathrm{mL}$, and their morphologies were taken under a microscope and a TEM. In addition, they were labeled with DiI and placed under a CLSM to take the morphologies of fluorescence. The particle size and potential of the two NPs were detected using a laser particle sizer.

We needed to detect the encapsulation rate and drug loading of the prepared NPs, so the standard curve of 10HCPT was constructed using high-performance liquid chromatography. The prepared NPs were split in methanol solution and added to a high-performance liquid chromatograph, their area under the curve was measured, and the entrapment efficiency and drug loading were calculated by the standard curve.

\section{Cell Culture and Establishment of an}

\section{Animal Xenograft Model}

Hepatoma SK-Hep1 cells were adherently cultured with MEM complete medium (10\% fetal bovine serum). ${ }^{30}$ The cells were all cultured in a cell incubator at $37{ }^{\circ} \mathrm{C}, 5 \%$ $\mathrm{CO} 2$, and $95 \%$ humidity and passaged or frozen when the cells were in the logarithmic phase. BALB/c nude mice (male, 4-5 weeks, 16-18 g/mouse) were purchased from the Animal Experiment Center of Chongqing Medical University, housed in SPF-grade cages, and maintained in accordance with guidelines set by the Animal Care Committee of Chongqing Medical University and approved by the Committee. SK-Hep1 cells were cultured until the logarithmic growth phase, the cell concentration was adjusted to $5-6 \times 10^{6} / \mathrm{mL}$, and $200 \mu \mathrm{L}$ of cells was subcutaneously injected into the root of the right hind leg of each mouse. ${ }^{32}$ Approximately 40 days later, the size of the subcutaneous tumor was approximately $0.6 \mathrm{~cm}^{3}$.

\section{Establishment of 3D Multicellular Tumor Sphere (MCTS) Model}

The 3D MCTSs were constructed following a previously reported gravity suspension method. ${ }^{35,36}$ SK-Hep1 cell concentration was adjusted to $5 \times 10^{4} / \mathrm{mL}$. A $10 \mathrm{~cm}$ cell culture flat bottom dish was opened, $30 \mu \mathrm{L}$ of cells were sucked up and dropped on the lid of the culture dish, approximately $20 \mathrm{~mL}$ of PBS solution was added to the culture dish to ensure humidity, and the culture dish was gently placed in the cell incubator for 15 days.

\section{In vitro Acoustic Phase Transition of NPs}

The dilution concentration of NPs was adjusted to $500 \mathrm{mg} /$ $\mathrm{mL}$, LIFU was turned on, the parameters were adjusted to pulse mode ( $2 \mathrm{~s}$ on, $2 \mathrm{~s}$ off), the power was $3 \mathrm{w} / \mathrm{cm}^{2},{ }^{32}$ the NPs were loaded into a $5 \mathrm{~mL}$ EP tube, placed in the center of the LIFU probe, irradiated with a focal length of $1.5 \mathrm{~cm}$, and the NPs were aspirated and dropped on a glass slide at $1 \mathrm{~min}$ and $5 \mathrm{~min}$ of irradiation and placed under a light microscope for observation.

\section{In vivo and in vitro PA/US Imaging of NPs}

PA imaging of NPs was first performed by full-band scanning with a wavelength of $680-970 \mathrm{~nm}$, and the wavelength with the strongest PA signal was selected as the experimental wavelength. The NPs were diluted at different concentrations and injected into the gel model holes. ${ }^{22}$ The acquired PA images were measured for intensity by a PA imager. At the 
same time, at the concentration of the most intense PA signal, NPs were given LIFU irradiation to collect the enhanced ultrasound images.

To perform in vivo PA imaging, subcutaneous xenografts have been previously constructed. The tumorbearing nude mice were divided into two groups, and then gas anesthesia was performed. The preimages without injection of NPs were collected, and then two kinds of NPs were injected from the tail vein. The PA images of the two groups were collected and analyzed as the excitation wavelength at $1 \mathrm{~h}, 3 \mathrm{~h}, 6 \mathrm{~h}$, and $24 \mathrm{~h}$ after injection.

\section{Targeting Efficiency of NPs in vitro}

The concentrations of SK-Hep1 and L02 cells (L02 is normal hepatocytes) were adjusted to $5 \times 10^{4} / \mathrm{mL}$, seeded in $2 \mathrm{~cm}$ flat-bottom dishes, and placed in a cell incubator for $24 \mathrm{~h}$, discarding the supernatant. Serum-free medium containing DiI-labeled iRGD-ICG-10-HCPT-PFP-NPs and ICG-10-HCPT-PFP-NPs were added to each well, which was then placed in a cell incubator for $30 \mathrm{~min}$ and removed. The liquid in the dish was removed and washed with PBS 3 times. Then, 4\% paraformaldehyde was used to fix the cells for $10 \mathrm{~min}$, and the cells were washed three times. ${ }^{30}$ Finally, $100 \mu \mathrm{L}$ DAPI was added to stain the nucleus for $5 \mathrm{~min}$, and the cells were washed again with PBS. Finally, the dish was observed by a CLSM.

Intracellular fluorescence of individual targeted tumor cells was also detected by flow cytometry. The concentrations of SK-Hep1 and L02 cells were adjusted to $5 \times 10^{4}$ / $\mathrm{mL}$, seeded in a six-well plate, and placed in the cell incubator for culture for $24 \mathrm{~h}$. The prepared iRGD-ICG -10-HCPT-PFP-NPs and ICG-10-HCPT-PFP-NPs labeled with DiI were added to the wells of a six-well plate, while PBS was used as a control group, and the 6-well plate was placed in the cell incubator for $1 \mathrm{~h}$. The cells were digested with trypsin, resuspended in PBS, and sent for flow cytometry later.

\section{The Penetration Ability of NPs in vitro}

The 3D MCTS model was carefully removed from the cell incubator and carefully transferred to a 24-well plate. DiIlabeled iRGD-ICG-10-HCPT-PFP-NPs and ICG-10-HCPT -PFP-NPs were added to the wells loaded with 3D MCTS and placed in the cell incubator for $1 \mathrm{~h}^{30}$ The 3D MCTSs were transferred to a $2 \mathrm{~cm}$ flat-bottom dish, with a small amount of PBS left, sent to a CLSM for photography, and scanned at a thickness of $1 \mu \mathrm{m}$. Finally, 3D reconstruction was performed.

\section{Antiproliferative Ability of NPs in vitro}

The antiproliferative ability of the NPs in vitro was examined using the CCK-8 assay. First, the concentration of SK-Hep1 was adjusted to $5 \times 10^{3} / \mathrm{mL}$. The cells were added to a 96 well plate, and three accessory wells were set for each group, which were placed in the incubator for $24 \mathrm{~h}$. The cells were divided into the following groups: 1: control, 2: ICG-PFPNPs, 3: ICG-10-HCPT-PFP-NPs, 4: iRGD-ICG-PFP-NPs, 5: iRGD-ICG-10-HCPT-PFP-NPs, 6: LIFU, 7: ICG-PFP-NPs + LIFU, 8: ICG -10-HCPT-PFP -NPs + LIFU, 9: iRGD-ICGPFP-NPs + LIFU, and 10: iRGD-ICG-10-HCPT-PFP-NPs + LIFU. NPs prepared with serum-free medium were added and placed in an incubator (the control group contained only serum-free medium), and the LIFU-treated group was irradiated at $3 \mathrm{w} / \mathrm{cm}^{2}$ for $5 \mathrm{~min}$ after $1 \mathrm{~h}$ of nanoparticle addition. After another incubation for $24 \mathrm{~h}, 10 \mu \mathrm{L}$ CCK-8 kit was added to each well and then placed in the incubator for $1 \mathrm{~h}$. After incubation, the optical density (OD) at $450 \mathrm{~nm}$ was read on a microplate reader. Survival ratio $=(\mathrm{As}-\mathrm{Ab}) /(\mathrm{Ac}-$ $\mathrm{Ab}$ ) $\times 100 \%$. (As is the experimental group, $\mathrm{Ab}$ is the control group without NPs, Ac is the blank without cells and containing only CCK-8).

\section{The Proapoptotic and Killing Ability of NPs in vitro}

Flow cytometry was used to detect the apoptosis ability of NPs. The concentration of SK-Hep1 was adjusted to $5 \times$ $10^{4} / \mathrm{mL}$, added to six-well plates, and placed in the incubator for $24 \mathrm{~h}$. They were divided into the same groups as mentioned above. NPs prepared in serum-free medium were added separately and placed in the incubator, and the LIFU-treated group was irradiated $1 \mathrm{~h}$ later. Twentyfour hours later, the cells in the wells were digested with trypsin, resuspended in PBS, and sent to flow cytometry for detection of apoptosis.

The killing ability of NPs on tumor cells was detected by calcein green-AM/propidium iodide(PI) double staining. The concentration of SK-Hep1 was adjusted to $5 \times$ $104 / \mathrm{mL}$ and added to $2 \mathrm{~cm}$ flat-bottom dishes for 24 h. These cells were divided into the following groups: Control, LIFU, ICG-10-HCPT-PFP-NPs, iRGD-ICG-10HCPT-PFP-NPs, ICG-10-HCPT-PFP-NPs+LIFU, and iRGD-ICG-10-HCPT-PFP-NPs+LIFU. The AM-PI staining solution was prepared, and $10 \mu \mathrm{L}$ AM stock solution and $15 \mu \mathrm{L}$ PI stock solution were added to $5 \mathrm{~mL}$ PBS. ${ }^{37}$ The staining solution was added to each dish and then 
placed in the incubator for $30 \mathrm{~min}$, and the fluorescence was observed with a CLSM.

\section{NPs Targeting Ability in vivo}

To validate the targeting ability of NPs in vivo, tumorbearing nude mice had been previously constructed. The fluorescent probe DIR was used to label iRGD-ICG-10HCPT-PFP-NPs and ICG-10-HCPT-PFP-NPs $(500 \mathrm{mg} /$ $\mathrm{mL}$ ). The tumor-bearing nude mice were divided into iRGD-ICG-10-HCPT-PFP-NP and ICG-10-HCPT-PFPNP groups, and then gas anesthesia was performed. First, the preimages were collected, and then the two NPs were injected from the tail vein. Tumor-bearing nude mice were placed in a fluorescence operating console, and fluorescence images at $1 \mathrm{~h}, 3 \mathrm{~h}, 6 \mathrm{~h}$, and $24 \mathrm{~h}$ were collected. Afterwards, the tumor-bearing mice were sacrificed, their viscera and tumors were separated, and then fluorescence images were collected. ${ }^{37}$

\section{Therapeutic Efficacy and Safety of NPs in vivo}

The tumor-bearing nude mice were divided into the same groups as mentioned above. They were given $200 \mu \mathrm{L}$ of the corresponding NP solution by tail vein injection, and the control group was given PBS injection. LIFU irradiation was started 1 hour after injection and irradiated once a day for 10 days. Changes in body weight and tumor volume were recorded during this period and The tumor volume was calculated using the formula: tumor volume $=$ length $\times(\text { width })^{2} / 2$. The relative tumor volume was represented as $\mathrm{V} / \mathrm{V}_{0}\left(\mathrm{~V}_{0}\right.$ is the initial tumor volume before treatment).At the end of the treatment, each group was sacrificed, the tumor mass was separated, pathological sections were obtained, hematoxylin-eosin staining (H\&E) was performed, and terminal deoxynucleotidyl transferase (TdT) dUTP nick-end labeling (TUNEL) and proliferating cell nuclear antigen (PCNA) detection were performed to determine the effect on apoptosis and proliferation. The isolated viscera (the heart, liver, spleen, lung, and kidney) were stained with H\&E to assess the safety of NPs in vivo.

\section{Statistical Analysis}

Statistical analysis was performed using SPSS 25.0 statistical analysis software for statistical analysis. Enumeration data were expressed as the mean $\pm \mathrm{SD}$. Analysis of variance was used for comparisons between multiple sample parameters. The $t$-test was used for comparison between two parameters. $\mathrm{P}<0.05$ was used to determine that the difference was significant. $\mathrm{P}<0.01$ indicated that the difference was significant. $\mathrm{P}<0.001$ indicated a highly significant difference.

\section{Results and Discussion \\ Characterization of NPs}

We found that iRGD-ICG-10-HCPT-PFP-NPs were observed under a light microscope with a regular shape, good dispersion, and small overall particle size (Figure 1A), and the DiI-labeled NPs were observed under a CLSM, which was consistent with ordinary microscopy in a white light channel. A uniform distribution of red fluorescence was also observed under a red laser channel without significant agglomeration (Figure 1B and C). TEM showed that the shape of the NPs was regular (Figure 1D), the outer layer showed phospholipid bilayer encapsulation, the middle was dense PFP, and the overall structure was intact. The particle size of iRGD-ICG-10-HCPT-PFP-NPs was $298.4 \pm$ $10.42 \mathrm{~nm}$ with a potential of $-35.4 \pm 6.23 \mathrm{mV}$ (Figure $1 \mathrm{E}$ and F), PDI was $0.073 \pm 0.23$, and the particle size of ICG10 -HCPT-PFP-NPs was $238.1 \pm 12.45 \mathrm{~nm}$ with a potential of-21.7 $\pm 5.12 \mathrm{mV}$ (Figure $1 \mathrm{G}$ and $\mathrm{H}$ ), and the particle size was stable in 7 days. The encapsulation was calculated to be $45.53 \pm 2.31 \%$, and the drug loading was $4.79 \pm$ $0.45 \%$. Through the characterization of the NPs, we found that the shape of the NPs was regular, the surface and internal structure of the NPs could also be seen under $\mathrm{TEM}^{41}$ showing a phospholipid bilayer, and the dense PFP was surrounded in the middle. The nanoparticle structure we prepared was intact and encapsulated all materials well in it. By particle size detection, the particle size of iRGD-ICG-10-HCPT-PFP-NPs was approximately $300 \mathrm{~nm}$, and this size was able to pass through the tumor interstitial space $(380-700 \mathrm{~nm})$ better and to reach the surface of tumor cells. ${ }^{8,42}$ The potential is a negative charge, which can have a longer blood circulation time. ${ }^{43}$ Because of the modification of polyethylene glycol (PEG) by the film-forming material, PEG molecules form a protective hydrophilic layer, which helps to avoid recognition by the immune system, thereby reducing the uptake of PEG-coated nanoparticles by macrophages, allowing NPs to have a long circulation time in the body, and reducing the phagocytosis of the reticuloendothelial system in the body. ${ }^{10,44}$ Through the detection 


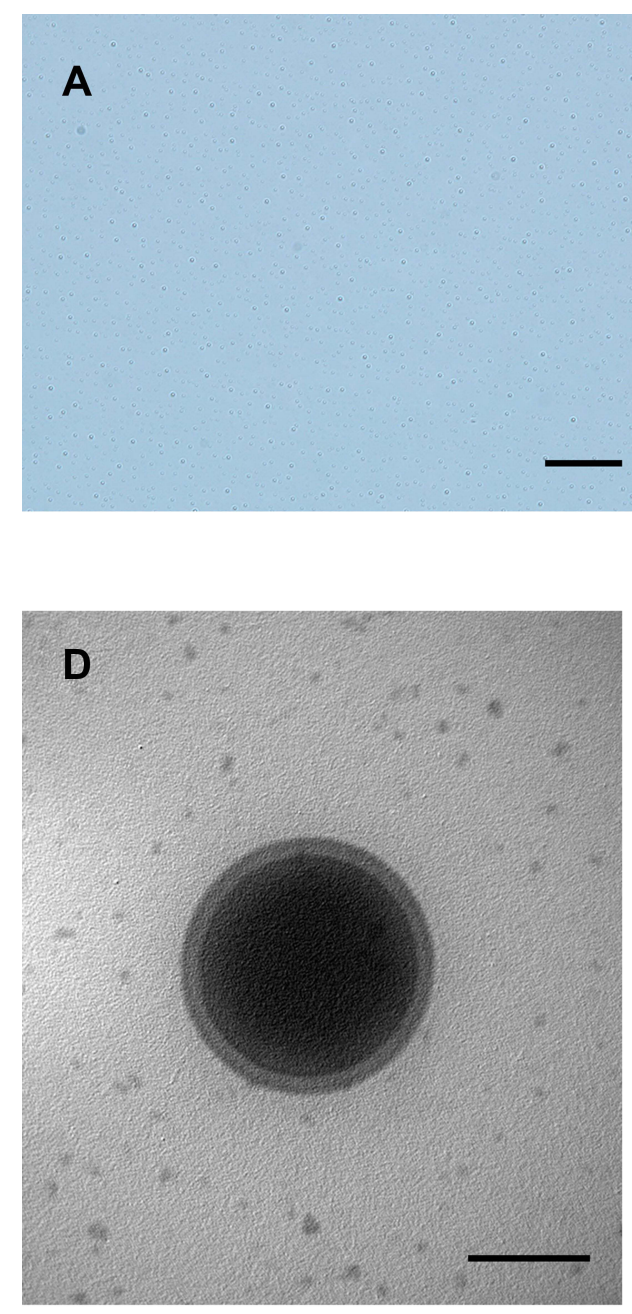

G

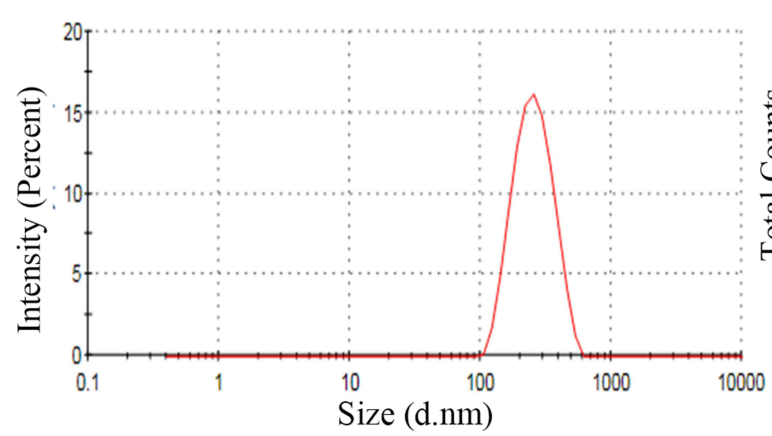

B
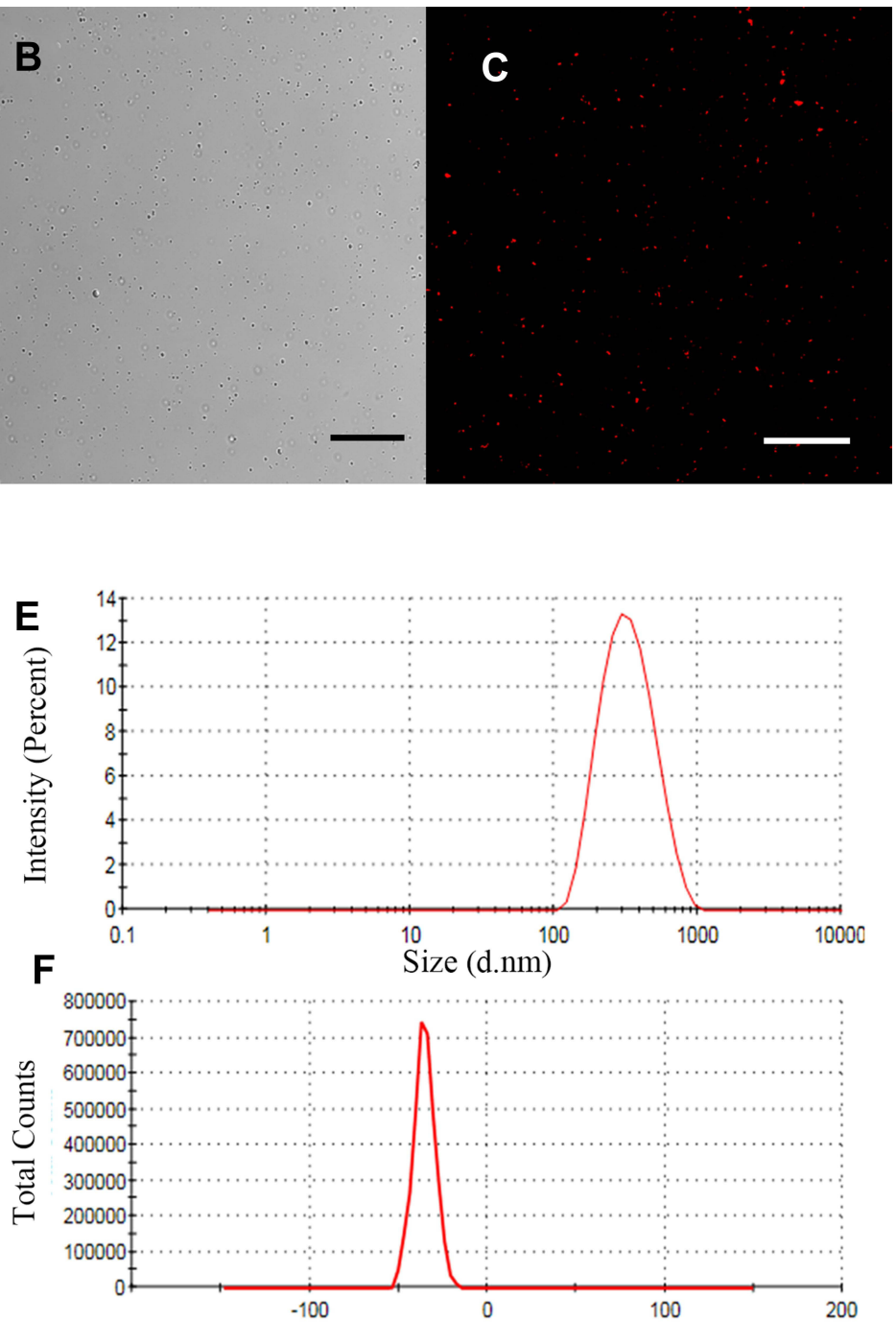

H Apparent Zeta Potential (mV)



Figure I In vitro characteristics of iRGD-ICG-10-HCPT-PFP-NPs and ICG-I0-HCPT-PFP-NPs (A) morphology of iRGD-ICG-I0-HCPT-PFP-NPs under a light microscope, scale bar: $20 \mu \mathrm{m}$ (B) morphology of iRGD-ICG-10-HCPT-PFP-NPs under the white light of a CLSM, scale bar: $20 \mu \mathrm{m}$ (C) morphology of iRGD-ICG-I0-HCPT-PFP-NPs under a red fluorescence channel of a CLSM, scale bar: $100 \mu \mathrm{m}$ (D) structure of iRGD-ICG-I0-HCPT-PFP-NPs under TEM, scale bar: $200 \mathrm{~nm}$ (E) particle size of iRGD-ICG -10-HCPT-PFP-NPs (F) zeta potential of iRGD-ICG-I0-HCPT-PFP-NPs (G) particle size of ICG-I0-HCPT-PFP-NPs (H) potential of ICG-I0-HCPT-NPs.

of a series of characterizations of the prepared NPs, we conclude that the NPs we prepared are regularly round in shape, have a small overall particle size, can better circulate in the body and enter the site of tumor tissue, and can perform subsequent in vitro and in vivo performance detection experiments. 


\section{In vitro Imaging and Phase Transition of NPs}

We observed that iRGD-ICG-10-HCPT-PFP-NPs were all able to produce PA signals at different concentrations of dilution, and the PA signals grew with increasing concentration (Figure 2A). The region of interest was selected on the PA detector to determine the PA signal intensity, and it could also be found that only a weak PA signal was visible in the sample with a concentration of $10 \mu \mathrm{g} / \mathrm{mL}$. As the concentration of ICG increases, the overall PA signal is linearly enhanced (Figure 2B). In the in vitro phase transition experiment of NPs, we first observed under a light microscope that the NPs presented better stability without

\section{A}

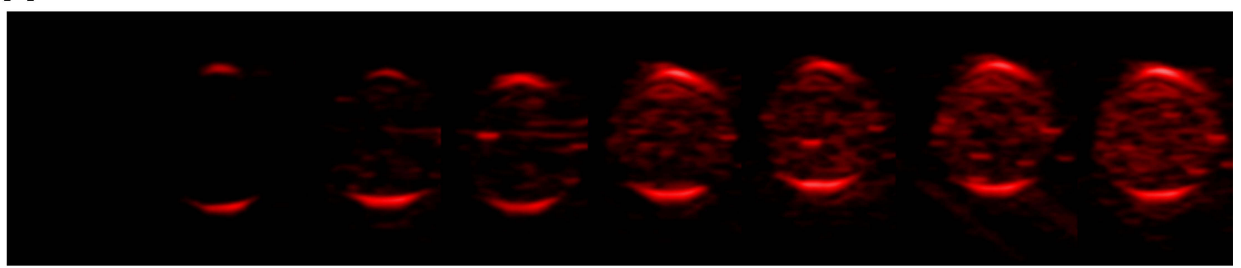

Control $\quad 10 \mu \mathrm{g} / \mathrm{ml} \quad 25 \mu \mathrm{g} / \mathrm{ml} \quad 50 \mu \mathrm{g} / \mathrm{m} \quad 100 \mu \mathrm{g} / \mathrm{ml} \quad 125 \mu \mathrm{g} / \mathrm{ml} \quad 250 \mu \mathrm{g} / \mathrm{ml} \quad 500 \mu \mathrm{g} / \mathrm{ml}$

B

C
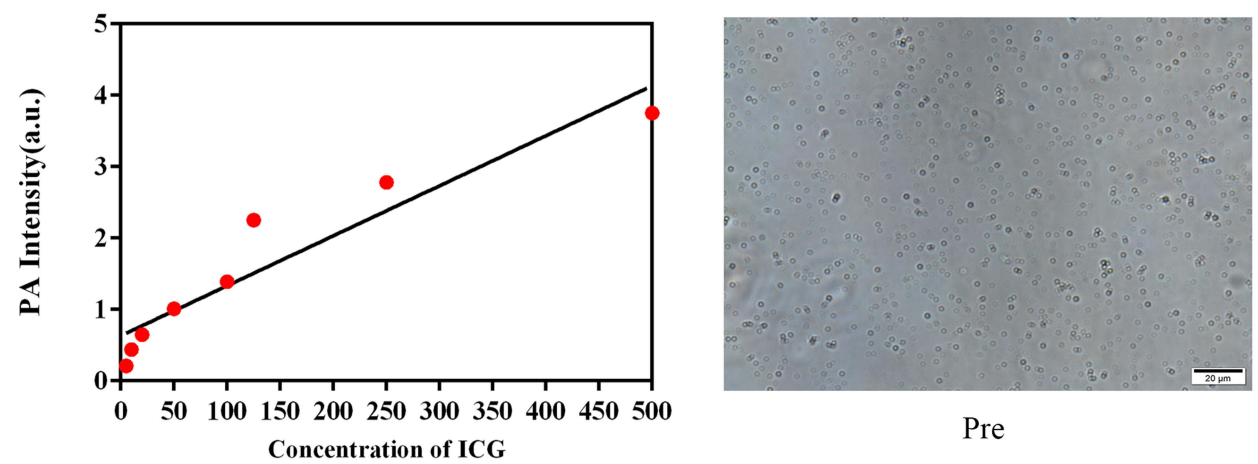

Pre

D

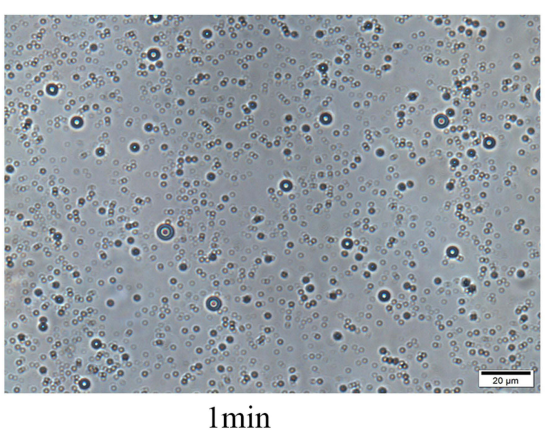

E

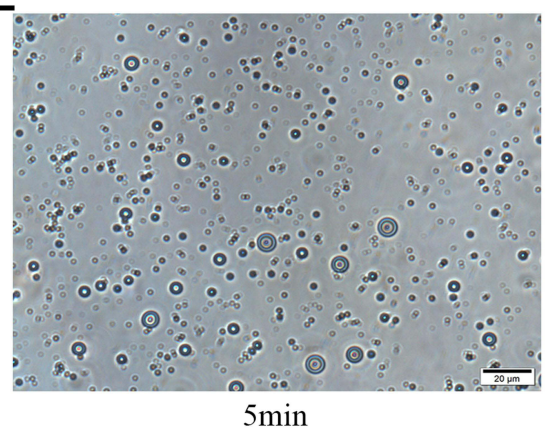

$F$

G

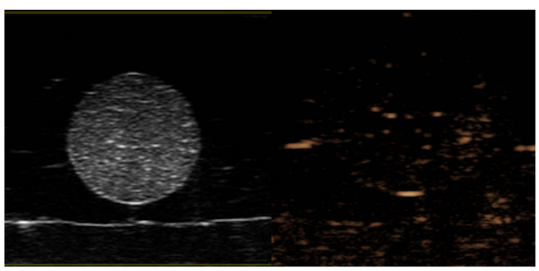

before

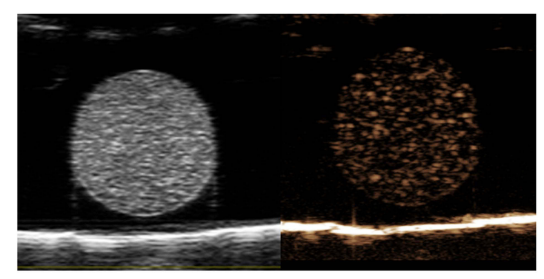

after

Figure 2 PA imaging and phase transition of NPs in vitro (A) PA images of iRGD-ICG-10-HCPT-PFP-NPs varying with ICG concentration in vitro (B) PA signal values of different concentrations measured by PA apparatus (C) images of NPs before LIFU irradiation (D and E) phase transition of NPs after LIFU irradiation, scale bar: $20 \mu$ m. (F) B-mode and enhanced ultrasound images of NPs before LIFU irradiation. (G) B-mode and enhanced ultrasound images of NPs after LIFU irradiation. 
any treatment, and the particle size was in a stable state (Figure 2C). After $1 \mathrm{~min}$ of LIFU irradiation, the NPs began to show a small part of the phase transition, as shown by the increase in the particle size of a small amount of NPs (Figure 2D). However, after $5 \mathrm{~min}$ of LIFU irradiation, the degree of phase transition of the NPs was more pronounced, more bulky NP distribution was observed throughout the field, and in practical observation, the NPs began to become unstable, showed four movements, and had ruptures (Figure 2E). Similar phase transitions can also be found after excitation with a laser. Additionally, NPs can produce enhanced ultrasound images under LIFU irradiation (Figure $2 \mathrm{~F}$ and $\mathrm{G}$ ).

As a light absorber, ICG is a near-infrared dye that is nearly harmless to the human body and easily metabolized. ${ }^{26,27,45}$ After loading the ICG package into NPs, they are excited by PA instruments to enable PA imaging, which is the basis for the ability of this nanoparticle to act as a molecular probe for PA imaging. Through experiments, we found that in in vitro PA imaging, NPs were able to produce PA signals at low ICG concentrations, and the resulting PA signals could be linearly enhanced as ICG concentrations increased, suggesting that the NPs were able to absorb light and to convert into PA signals. It has been documented that the sensitivity of PA imaging is better than that of computed tomography (CT), and this result is also of reference value for clinical practice. $^{46}$

Moreover, 10-HCPT encapsulated in NPs can play an antiproliferative and proapoptotic role; however, due to the direct effect in vivo, the drug cannot be accurately released, and there are many side effects when applied to the whole body. Encapsulating it into NPs and giving accurate release can reduce both systemic side effects and the dose of the drug used to play an improved therapeutic role. ${ }^{29,47}$ LIFU is a low-intensity focused ultrasound independently developed in our laboratory and is characterized by a small total energy, a small and precise irradiated area, and the ability to focus ultrasound energy to achieve precise irradiation. ${ }^{30,32,48}$ NPs were administered in vitro for LIFU irradiation, and it was observed that NPs that were otherwise small in size and regular in morphology showed varying degrees of phase transition and rupture over time after receiving LIFU irradiation. This finding is because through the irradiation of LIFU, the encapsulated PFP in the NPs produces a phase transition, which is converted from liquid to gas, so that the NPs enlarge, thereby enhancing their ability for US imaging while triggering the rupture of the NPs and releasing the 10-HCPT encapsulated in them, playing a role in releasing the drug.

\section{In vitro Targeting of NPs}

SK-Hep1 highly expressed the NRP-1 receptor, and we applied two assays to target NPs in vitro (Expression of NRP-1 by individual cell lines is described in the Supplemental Material). Under a CLSM (Figure 3), blue fluorescence was emitted by the nucleus, and red fluorescence was emitted by DiI-labeled NPs. We found that in the SKHepl group, red fluorescence could be seen around the nucleus in the DiI-labeled iRGD-ICG-10-HCPT-PFP-NP group, and after passing through Merge, red fluorescence could be found to focus around the blue fluorescence, suggesting that the NPs have the ability to target tumor cells. In the DiI-labeled ICG-10-HCPT-PFP-NP group, only blue fluorescence emitted from the nucleus was observed, and no more significant red fluorescence was observed around the cells. However, in the L02 group, in both the DiI-labeled iRGDICG-10-HCPT-PFP-NP group and the DiI-labeled ICG-10HCPT-PFP-NP group, only blue fluorescence emitted from the nucleus was observed, and no red fluorescence was observed around the cells. These results suggest that NPs equipped with the iRGD peptide have strong targeting ability and no obvious targeting ability to normal hepatocytes. ${ }^{32}$ Similar results were found by measuring the fluorescence intensity inside the cells by flow cytometry (Figure 4A). In the SK-Hep1 group, the intracellular fluorescence intensity of the DiI-labeled iRGD-ICG-10-HCPT-PFP-NP group was significantly higher than that of the DiI-labeled ICG-10-HCPTPFP-NP group, and the difference was highly significant $(\mathrm{P}<0.001)$. In the L02 group, no significant fluorescence was observed in the two groups, and the difference was not statistically significant $(\mathrm{P}>0.05)$. No significant intracellular fluorescence was observed in the control group, also suggesting better targeting of iRGD NPs (Figure 4B).

NPs are equipped with the iRGD peptide, a small polypeptide called a tumor-homing peptide. This peptide is able to target tumor cells, and, through its attachment to the NRP1 receptor on the surface of tumor cells, promotes the entry of the carried substance into the interior of tumor cells Hepatoma SK-Hep1 cells highly expressed the NRP-1 receptor as previously described, so these cells were selected for cell experiments. ${ }^{16-19}$ From the CLSM results, in hepatoma cells, after coincubation of iRGD-ICG-10-HCPT-PFPNPs, NPs significantly accumulated around tumor cells. The 


\section{SK-Hep 1}

iRGD-ICG-10-HCPT-PFP-NPs ICG-10-HCPT-PFP-NPs
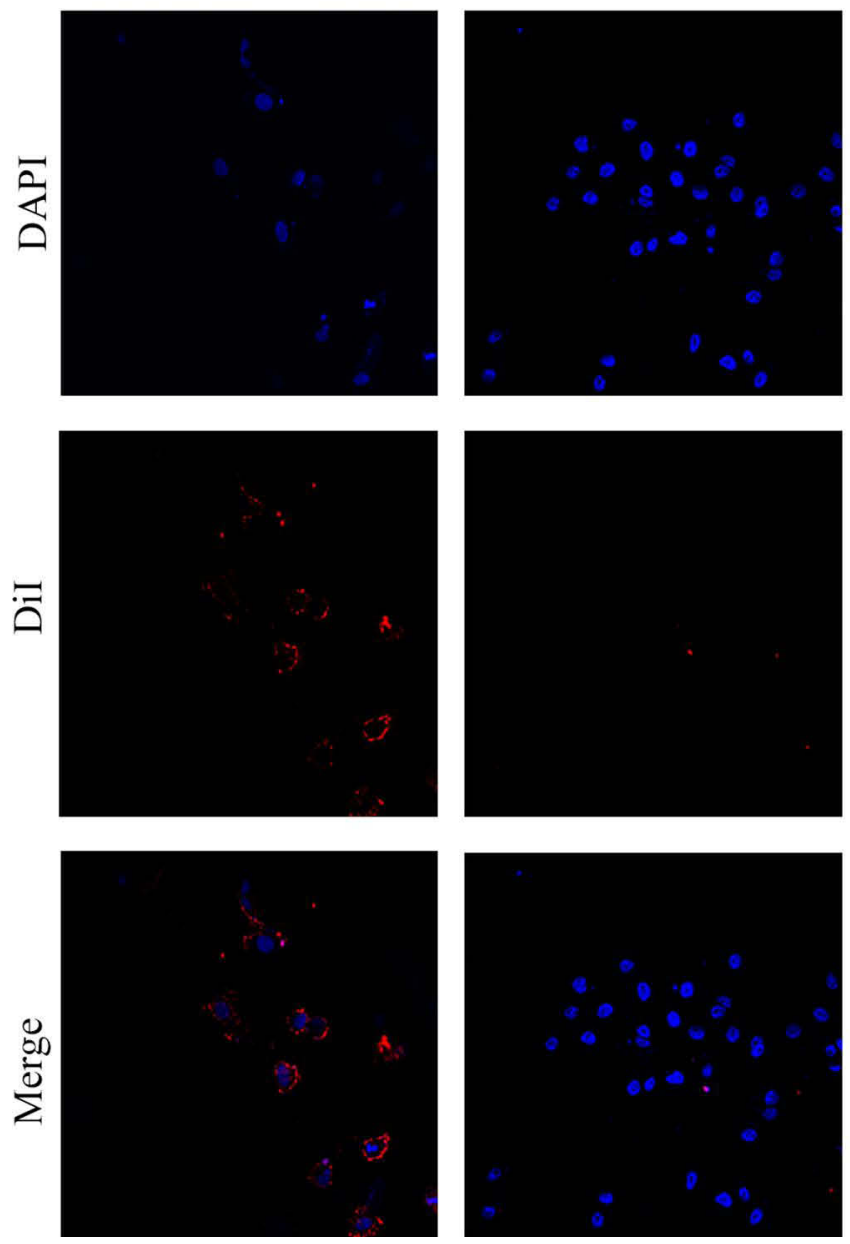

L02
iRGD-ICG-10-HCPT-PFP-NPs
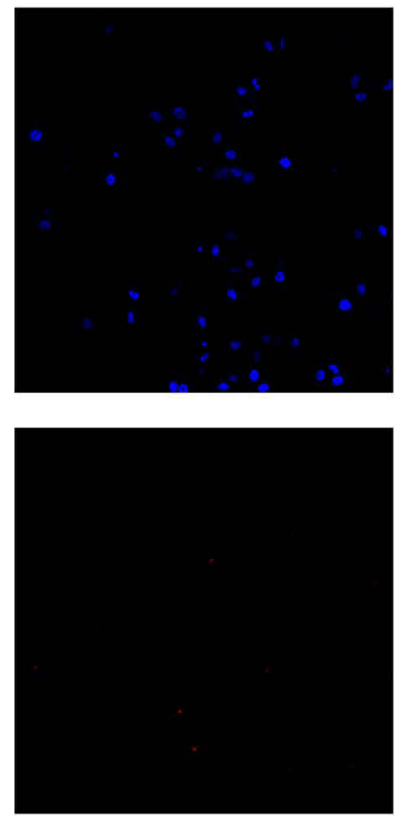

ICG-10-HCPT-PFP-NPs
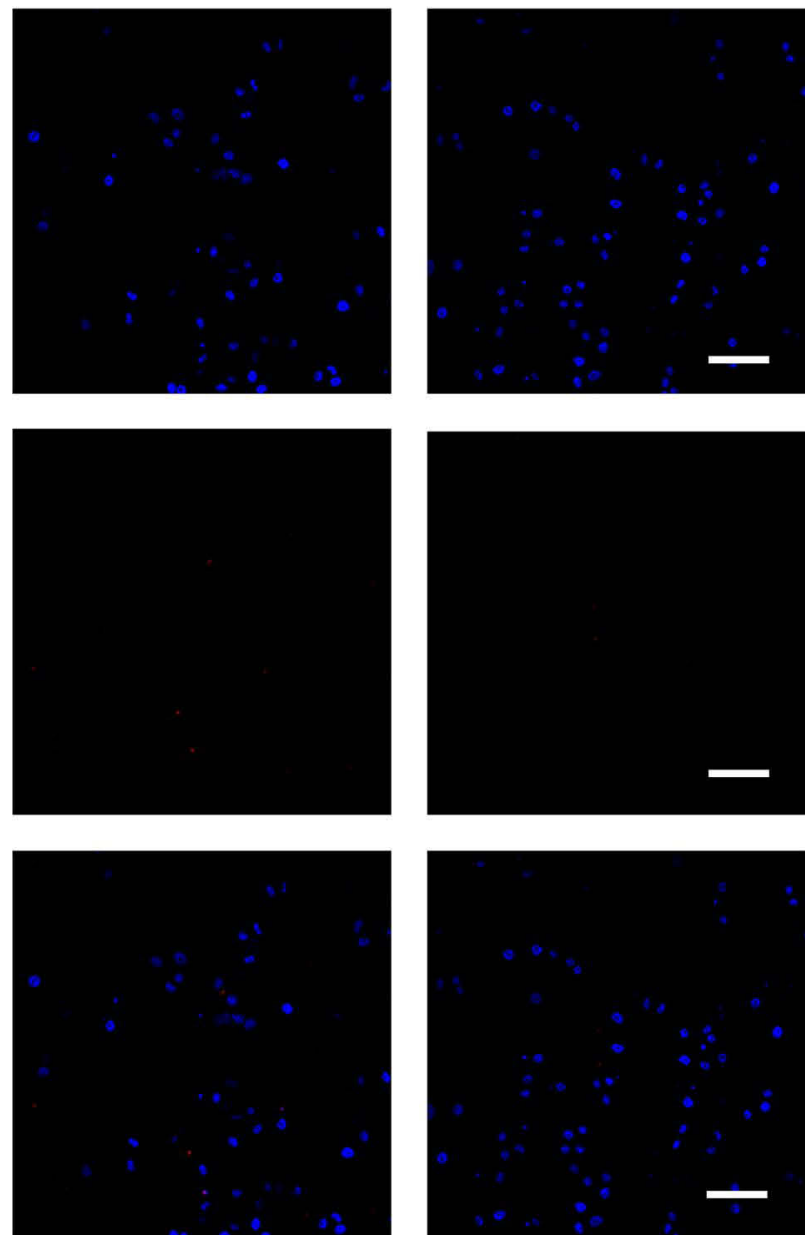

Figure 3 iRGD-ICG-I0-HCPT-PFP-NPs and ICG-I0-HCPT-PFP-NPs were used for target-finding experiments with SK-HepI cells and L02 cells, respectively, as observed under a CLSM, scale bar: $100 \mu \mathrm{m}(\mathrm{n}=3)$.

A

Control
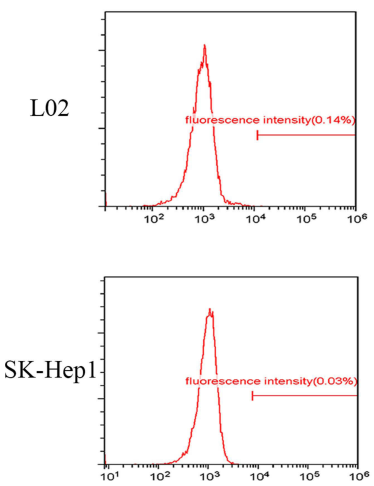

ICG-10-HCPT-PFP-NP
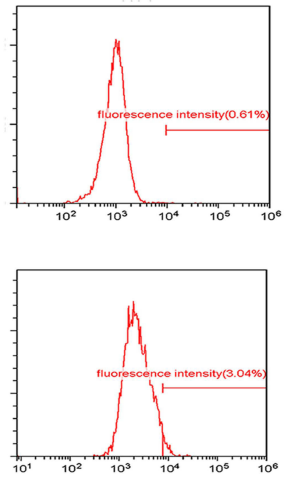

iRGD-ICG-10-HCPT-PFP-NP
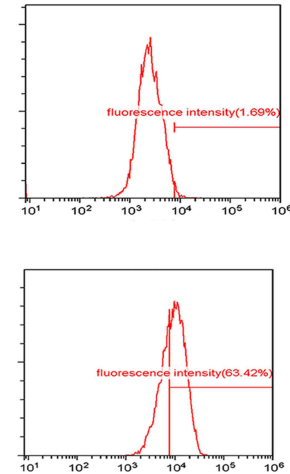

B

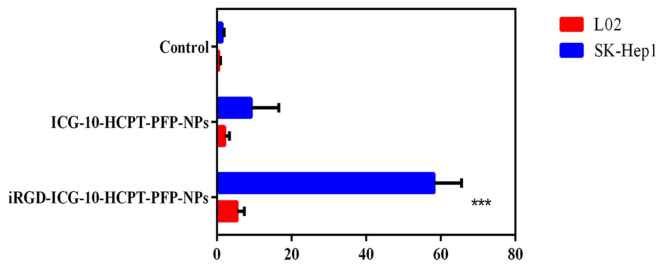

fluorescence intensity $(\%)$

Figure 4 Detection of the target-finding ability of NPs by flow cytometry. (A) iRGD-ICG-10-HCPT-PFP-NPs and ICG- I0-HCPT-PFP-NPs were used in target-finding experiments with SK-Hepl cells and L02 cells, respectively, and fluorescence was detected by flow cytometry $(n=5)(B)$ statistics of fluorescence intensity in each group, $* * * P<0.001$. 
results demonstrated that iRGD-ICG-10-HCPT-PFP-NPs are targeted to tumor cells with good targeting, which lays a foundation for the future use of NPs in the treatment of tumors. At the same time, the same conclusion was reached when the intensity of intracellular fluorescence was detected by flow cytometry, again demonstrating the targeting of iRGD-ICG-10-HCPT-PFP-NPs.

\section{Killing Effect of NPs on Tumor Cells}

Stained SK-Hepl cells were excited by emitting two wavelengths of lasers by a CLSM, where green fluorescence represents surviving cells and red fluorescence represents dead cells. ${ }^{37}$ We found that only green fluorescence was emitted in the control group, suggesting that all cells survived (Figure 5A). When ICG-10-HCPT-PFP-NPs and iRGD-ICG -10-HCPT-PFP-NPs were added alone, a small amount of red fluorescence was observed, and most of them still exhibited green fluorescence, suggesting that only a small part of the cells died (Figure 5B and C).In the LIFU irradiation alone group, the results were similar to those of the previous two groups (Figure 5D). However, in the ICG-10-HCPT-PFP-NPs + LIFU group, more red fluorescence was observed, which was generally similar to green fluorescence, suggesting that cell death and survival were close, and the number of surviving cells was less than that in the previous three groups (Figure 5E). In the iRGD-ICG-10-HCPT-PFP-NPs+LIFU group, a large amount of red fluorescence and only a very small amount of green fluorescence were seen throughout the field, suggesting that a large number of cells died overall and that only a small number of cells survived (Figure 5F). Tumor cells were killed more after administration of iRGD-ICG-10HCPT-PFP-NPs and irradiation with LIFU.

\section{Penetration Ability of NP to Tumor Cells} We judged the penetration depth of NPs by the red fluorescence emitted by DiI under a CLSM. We found

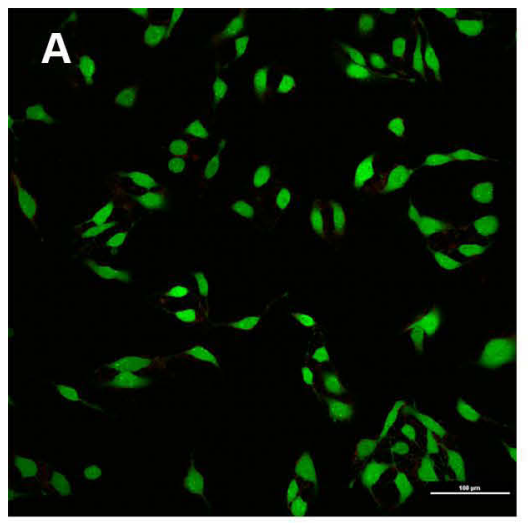

Control

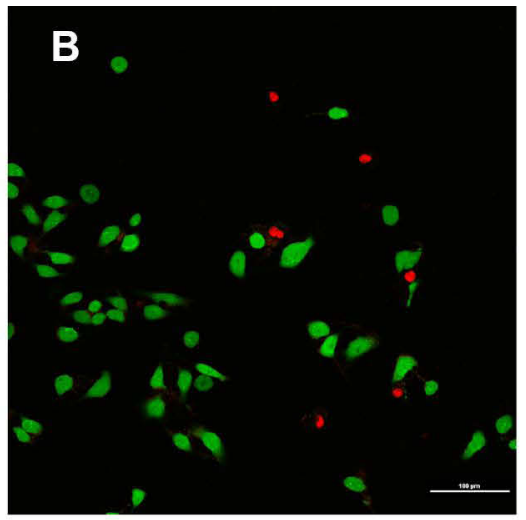

ICG-10-HCPT-PFP-NPs

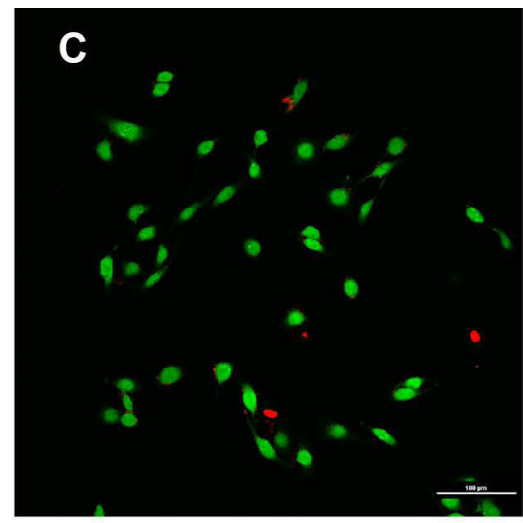

iRGD-ICG-10-HCPT-PFP-NPs
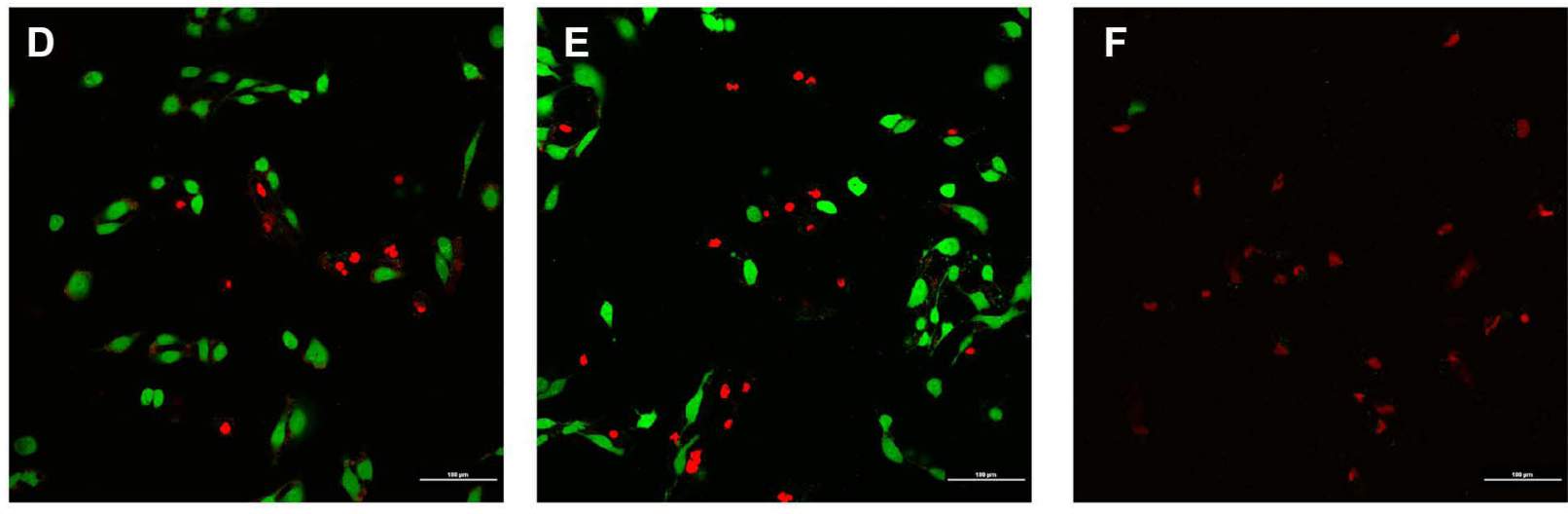

LIFU

ICG-10-HCPT-PFP-NPs+LIFU

iRGD-ICG-10-HCPT-PFP-NPs+LIFU

Figure $\mathbf{5}$ The killing effect of NPs on hepatoma cells in vitro was detected by AM/PI double staining and observed under a CLSM: (A) control, (B) ICG-I0-HCPT-NPs, (C) iRGD-ICG-I0-HCPT-NPs, (D) LIFU, (E) ICG-I0-HCPT-NPs+LIFU,and (F) iRGD-ICG-I0-HCPT-NPs+LIFU; scale bar: $100 \mu \mathrm{m}$.(n=5). 
that after 3D reconstruction by a CLSM, the overall MCTS had a spherical shape with a diameter of approximately $100 \mu \mathrm{m} .{ }^{30}$ At the layer-scanned interface, it was found that in the iRGD-ICG-10-HCPT-PFP-NP group, the overall penetration depth of DiI-labeled NPs was up to $49.34 \mu \mathrm{m}$, and there was much red fluorescence at the edge and inside of the MCTS as a whole (Figure 6A). In the ICG-10-HCPT-PFP-NP group, the penetration depth was $24.39 \mu \mathrm{m}$, and the red fluorescence at the edge of the MCTS was weak (Figure 6B). The penetration depth of the group loaded with iRGD peptide was twice that of the group without peptide, suggesting that NPs loaded with iRGD peptide have a strong targeting and penetration ability for tumor cells. In addition to targeting tumor cells, iRGD peptides also promote the penetration of the carried substances into the tumor cell membrane and deep into the cell interior. We constructed a 3D MCTS, a cell environment that has the advantage of being more stereoscopic and closer to the in vivo tumor growth environment than cells commonly, thus also better verifying the penetration ability of iRGD peptides. ${ }^{36}$ This finding laid the foundation for subsequent treatment, especially when chemotherapeutic drugs were released after LIFU irradiation rupture, which could play a deeper role in releasing drugs to achieve a better therapeutic effect.

\section{Proapoptotic Ability of NPs on Tumor Cells}

We verified the proapoptotic effect of NPs with different treatment modalities on SK-Hep1 cells by flow cytometry (Figure 7A). We found that the early and late apoptotic rates were the highest in the iRGD-ICG-10-HCPT-PFPNPs+LIFU group and the difference was highly significant $(\mathrm{P}<0.001)$. In addition, the iRGD-ICG-PFP-NPs + LIFU group was second highest and the difference was significant $(\mathrm{P}<0.01)$. The rates of both of these groups were higher than those in the group without LIFU irradiation and supplemented with the same NPs, and the other groups given LIFU irradiation had little difference in the overall early and late apoptotic rates between the non-LIFU irradiation group. The apoptotic rate was also not very different between the control group and the LIFU irradiation alone group. The experimental results suggested that the iRGD-ICG-10-HCPT-PFP-NPs+LIFU group had the strongest proapoptotic effect on tumor cells (Figure 7B).

\section{Antiproliferative Ability of NPs Against Tumor Cells}

We found that in the iRGD-ICG-10-HCPT-PFP-NPs + LIFU group, the cells had the lowest survival rate, the strongest antiproliferative ability, and greater toxicity to the cells, and the difference was highly significant $(\mathrm{P}<0.001)$ (Figure 7C). Second, the iRGD-ICG-PFP-NPs + LIFU group had lower cell survival than the group supplemented with the same NPs without LIFU irradiation. However, in the remaining LIFU-treated and nonLIFU-irradiated groups, the cell survival rate was similar and higher than that in the previous two groups. Cell survival was highest in the control group and the LIFU irradiation alone group, with no significant difference. These results suggest that the antiproliferation ability of cells is the strongest in the presence of iRGD-ICG-10HCPT-PFP-NPs + LIFU. We performed three experiments to examine the killing effect of NPs as well as the ability to resist proliferation and to promote apoptosis in vitro, and we found that the iRGD-ICG-10-HCPT-PFP-NPs + LIFU group was the best. It is concluded that after iRGD-ICG-10-HCPT-PFP-NPs actively target tumor cells, they penetrated deeply into the tumor interior, while undergoing phase transition and rupture by LIFU irradiation, produced a physical burst effect and released the carried chemotherapeutic drugs, which has a synergistic effect in vitro and can promote apoptosis and antiproliferation of tumor cells.

\section{Targeting of NPs in vivo}

Through the detection of fluorescence in vivo (Figure 8A), we found that no significant fluorescence signal was detected in either group when the NPs were not injected. In the first hour after injection, the iRGD-ICG-10-HCPTPFP-NP group showed a strong fluorescence signal in the liver, and a small amount of fluorescence signal was detected at the blood vessels between the liver and subcutaneous tumor, while in the ICG-10-HCPT-PFP-NP group, only the fluorescence signal was observed in the liver, and no significant fluorescence signal was observed at the other sites. At $3 \mathrm{~h}$ after injection, in the iRGD-ICG -10-HCPT-PFP-NP group, the fluorescence signal in the tumor area became stronger. However, in the ICG-10HCPT-PFP-NP group, there was still only a fluorescence signal in the liver region, and no more significant fluorescence signal was observed in the tumor region. At 6 hours after injection, the fluorescence signal in the tumor area 

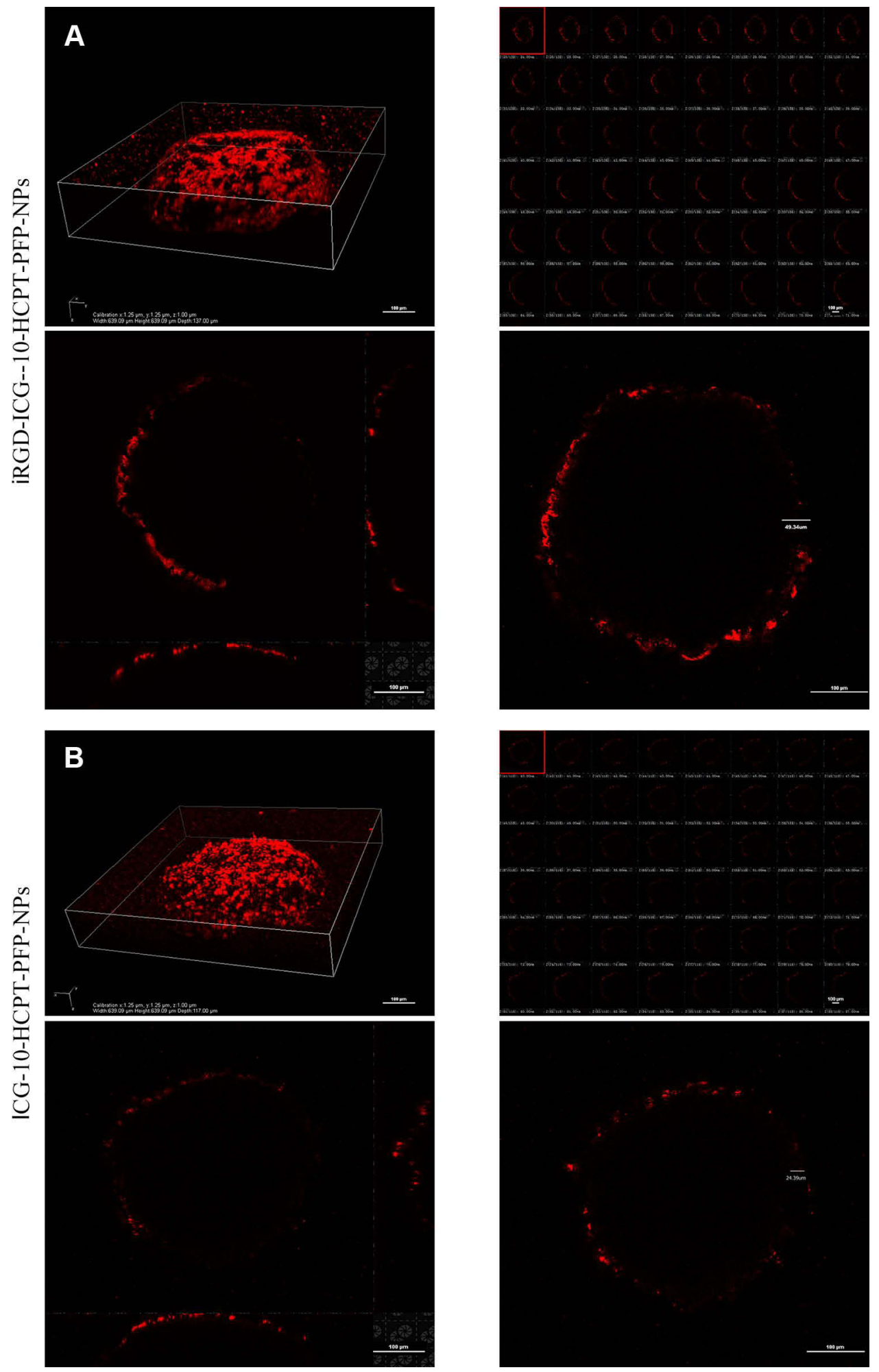

Figure 6 The penetration of NPs was detected by 3D MCTS. (A) iRGD-ICG-10-HCPT-PFP-NPs were coincubated with 3D MCTS and scanned at a thickness of I $\mu$ m per layer, and 3D reconstruction was performed; scale bar: $100 \mu \mathrm{m}$. (B) ICG-I0-HCPT-PFP-NPs were coincubated with 3D MCTSs and scanned at a thickness of I $\mu \mathrm{m}$ per layer, and 3D reconstruction was performed; scale bar: $100 \mu \mathrm{m} .(\mathrm{n}=3)$.

continued to increase in the iRGD-ICG-10-HCPT-PFP-NP group. In the ICG-10-HCPT-PFP-NP group, the fluorescence signal could still be observed only in the liver region, and after 24 hours it was the same as at 6 hours. The viscera and tumor mass were separated from the two groups of nude mice after sacrifice, and the detected 
A
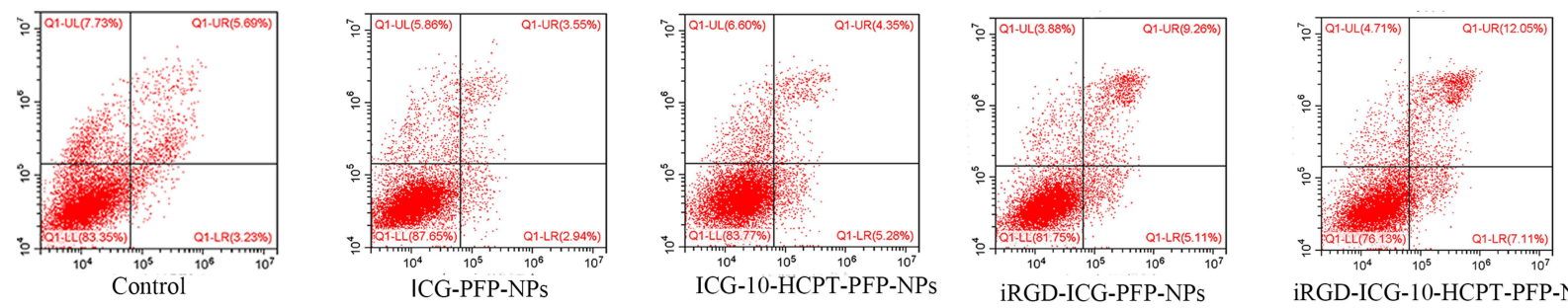

iRGD-ICG-10-HCPT-PFP-NPs

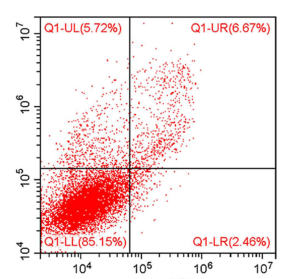

LIFU

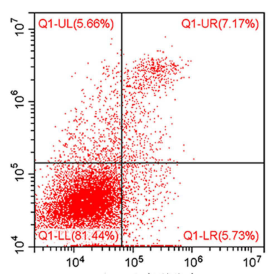

ICG-PFP-NPs + LIFU

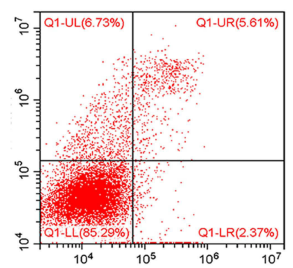

ICG-10-HCPT-PFP-NPs+LIF
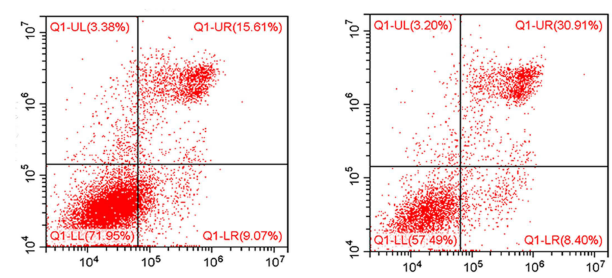

iRGD-ICG-10-HCPT-PFP-NPs+LIFU

B

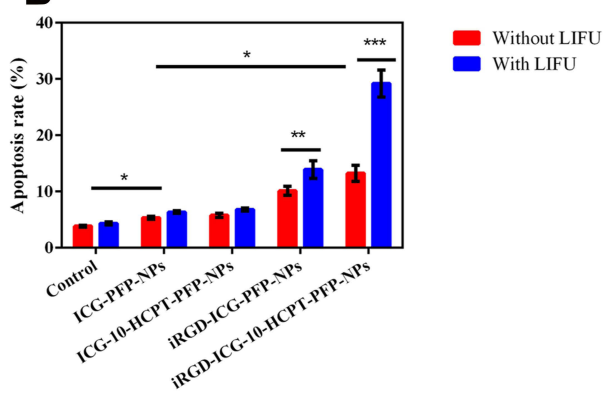

C

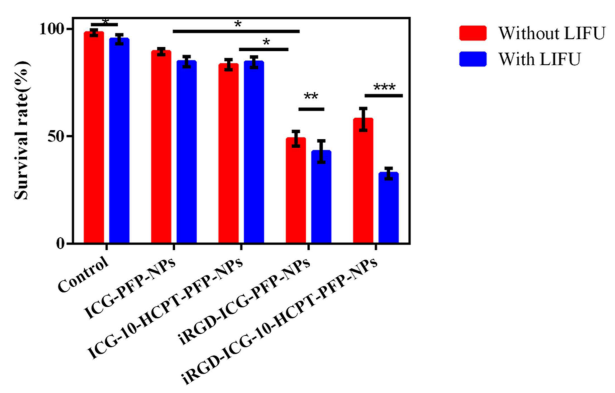

Figure 7 Detection of proapoptotic and antiproliferative properties of NPs $(\mathbf{A})$ apoptosis of HCC cells in each group detected by flow cytometry ( $\mathrm{n}=5)(\mathbf{B})$ statistics of flow cytometry results, ${ }^{*} \mathrm{P}<0.05,{ }^{*} * \mathrm{P}<0.0 \mathrm{I}, * * * \mathrm{P}<0.00 \mathrm{I}(\mathbf{C})$ anti proliferation detected by theCCK-8 assay, $* \mathrm{P}<0.05,{ }^{*} * \mathrm{P}<0.0 \mathrm{I}$, and $* * * \mathrm{P}<0.00 \mathrm{I}(\mathrm{n}=4)$.

fluorescence signals were consistent with those in vivo. This finding was confirmed by counting the fluorescence signal intensity at each time point (Figure 8B).

We found similar results on PA imaging of tumorbearing nude mice (Figure $8 \mathrm{C}$ ). No significant PA signal was detected in the tumor area of nude mice in either group before injection of NPs. At 1 hour after injection, a small amount of PA signal began to appear in the iRGDICG-10-HCPT-PFP-NP group, but no significant PA signal was detected in the ICG-10-HCPT-PFP-NP group. Over time, after $3 \mathrm{~h}$, the PA signal gradually increased in the iRGD-ICG-10-HCPT-PFP-NP group, and only a small amount of PA signal was detected at the edge of the tumor area in the ICG-10-HCPT-PFP-NP group. After 6 hours, the PA signal remained in the iRGD-ICG-10-HCPTPFP-NP group, and a small amount of PA signal appeared in the ICG-10-HCPT-PFP-NP group, and after 24 hours it was the same as at 6 hours. This finding was confirmed by counting the intensity of the PA signal at each time point in the tumor area (Figure 8D). At the same time, two groups were given ultrasonic detection, and the following results were obtained. No significant contrast-enhanced ultrasound (CEUS) signal was detected in the tumor area of either group before nanoparticle injection. At 1 hour after injection, after irradiation with the two groups of LIFU, more CEUS signals appeared in the iRGD-ICG-10HCPT-PFP-NP group, while no significant CEUS signals were detected in the ICG-10-HCPT-PFP-NP injection group (Figure 8E).

We constructed a subcutaneous xenograft model of SKHep1 to simulate the situation of tumors in vivo and continued to verify the performance of NPs with experiments in vivo. This result indicated that iRGD-ICG-10-HCPTPFP-NPs also had equally good targeting in vivo and were able to reach the tumor site more quickly than ICG-10-HCPT -PFP-NPs, thereby increasing enrichment in the tumor area. This finding is also mediated by the excellent targeting of tumor homing peptides. ${ }^{17,49}$ In PA imaging experiments in vivo, we found the same results. This experiment demonstrates the ability of NPs to perform PA imaging in vivo, and 

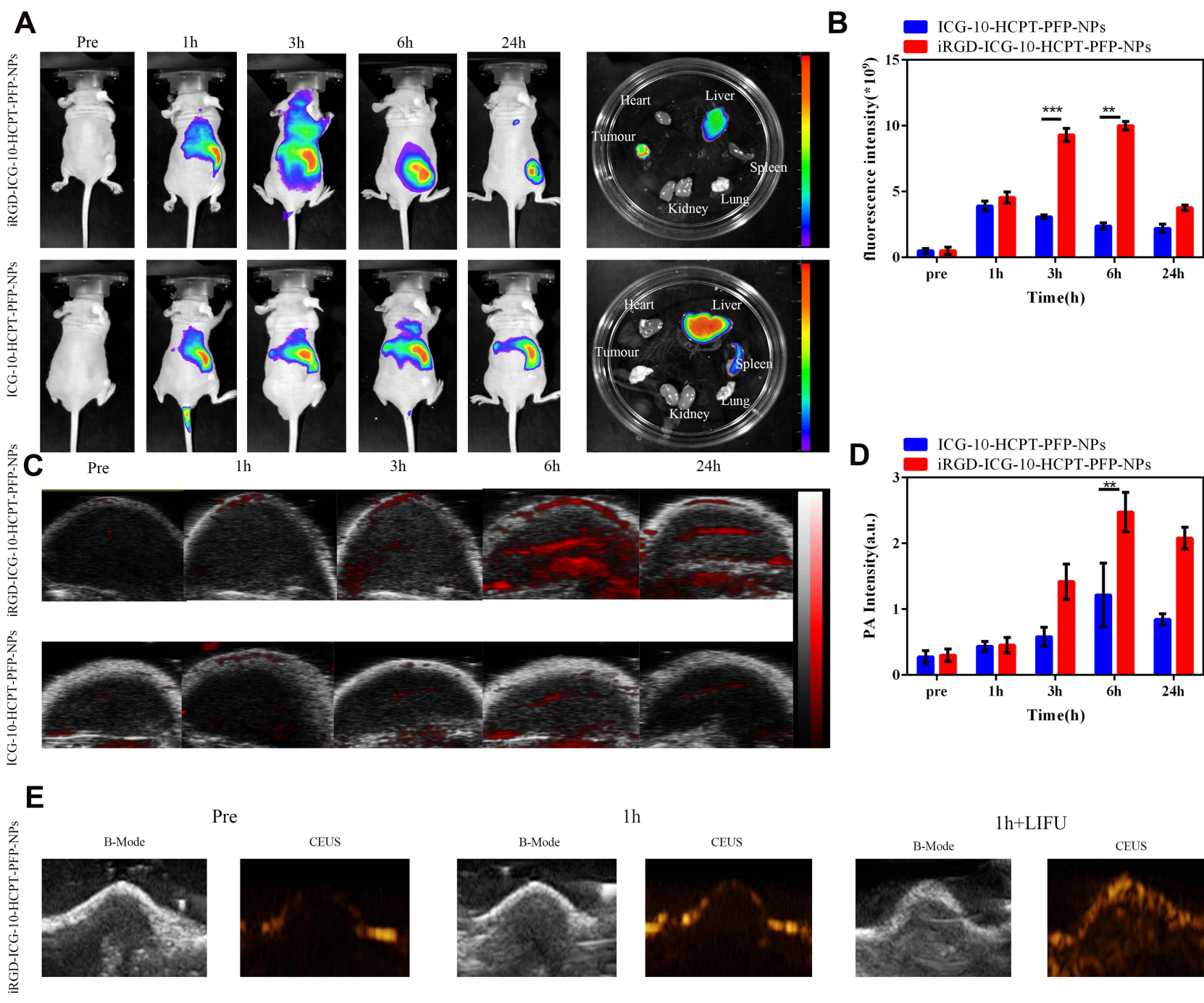

Pre

$1 \mathrm{~h}$
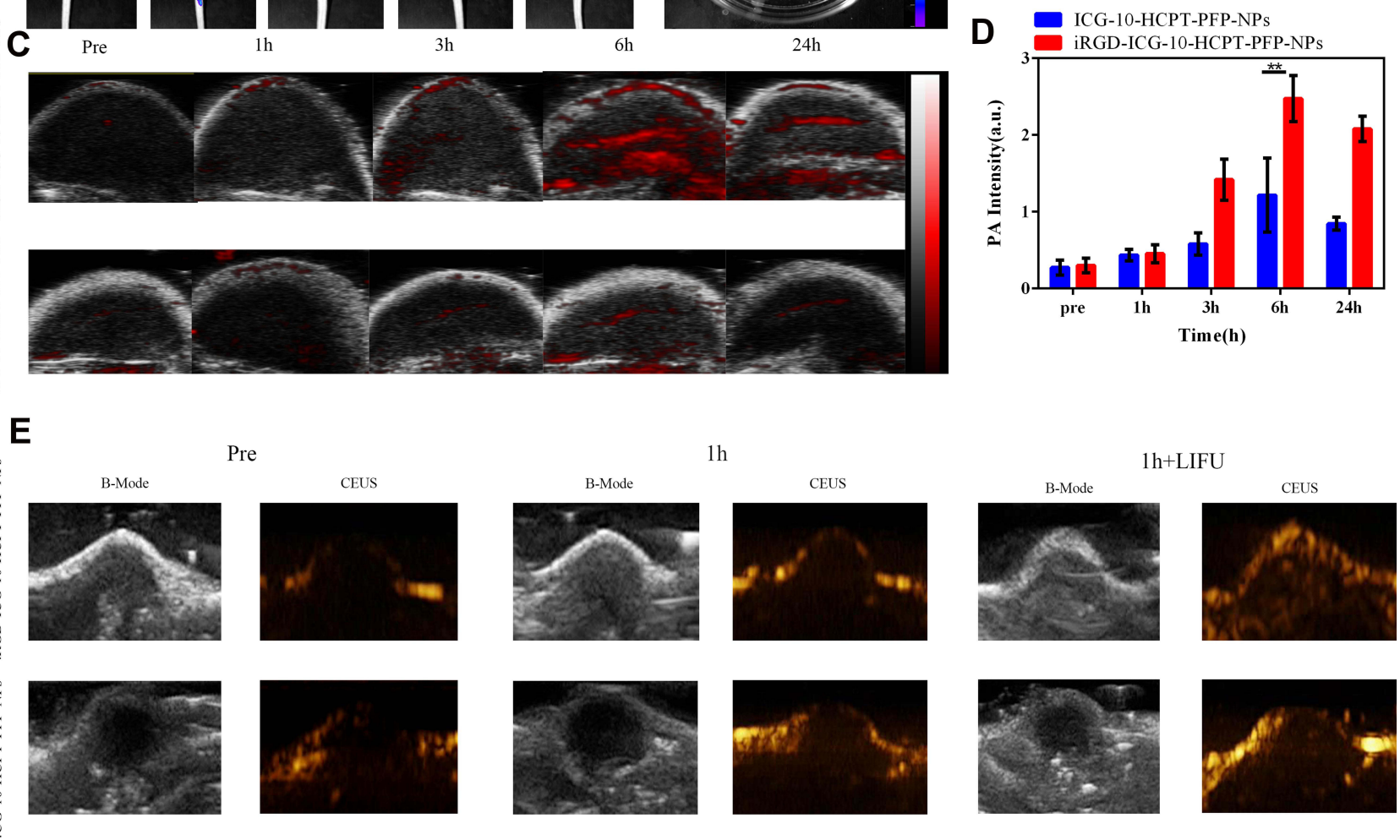

$\mathrm{h}+\mathrm{LIFU}$

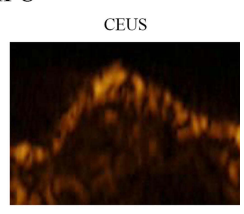

Figure $8 \mathrm{FL}$ and PA/US imaging of Nps in vivo. (A) In vivo fluorescence as well as ex vivo organ fluorescence images in the iRGD-ICG-I0-HCPT-PFP-NP and ICG-I0-HCPTPFP-NP groups. (B) Fluorescence intensity statistics of tumor sites, $* * P<0.01$ and $* * * P<0.00 I(n=3)$. (C) PA signal intensity statistics of tumor sites in the iRGD-ICG-I0HCPT-PFP-NP and ICG-I0-HCPT-PFP-NP groups. (D) PA signal intensity statistics of tumor sites, **P<0.0I, $(\mathrm{n}=3)$. (E) B-mode ultrasound and enhanced CEUS ultrasound images of tumor sites in the iRGD-ICG-10-HCPT-PFP-NP and ICG-10-HCPT-PFP-NP groups before and after LIFU irradiation in vivo.

due to their strong targeting, they can quickly reach the tumor area, which also provides a basis for NPs to achieve early diagnosis of tumors. At the same time, in the US imaging experiments in vivo, no CEUS images appeared before the injection of iRGD nanoparticles. However, at 1 hour after injection, after LIFU irradiation, CEUS images of the iRGDICG-10-HCPT-PFP-NP group were visible. Additionally, the targeting of iRGD-ICG-10-HCPT-PFP-NPs in vivo and the ability to enhance ultrasound imaging under LIFU irradiation were demonstrated.

\section{Therapeutic Effect of NPs in vivo}

After treating the tumor-bearing nude mice in different groups, we observed the following results (Figure 9A). There were no significant changes in body weight during the overall treatment time in either group (Figure 9B). The iRGD-ICG-10HCPT-PFP-NPs+LIFU group had the greatest tumor changes and the highest tumor inhibition rate by measuring the tumor size and sacrificing the nude mice and isolating the tumor after the end of treatment, and the difference was highly significant $(\mathrm{P}<0.001)$. The iRGD-ICG-PFP-NPs + LIFU group was 


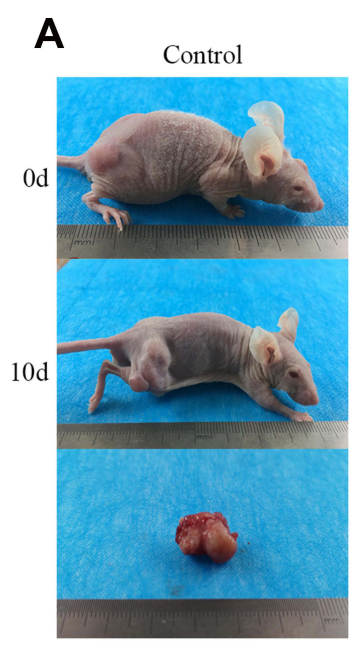

LIFU

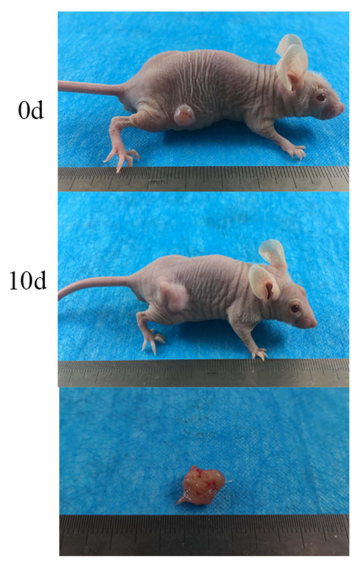

ICG-PFP

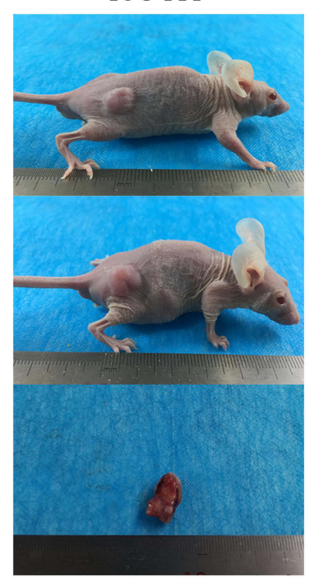

ICG-PFP+LIFU

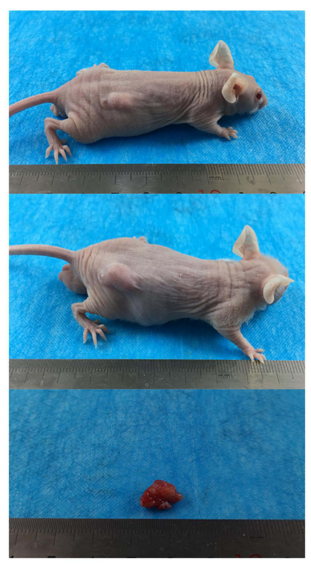

ICG-10-HCPT-PFP

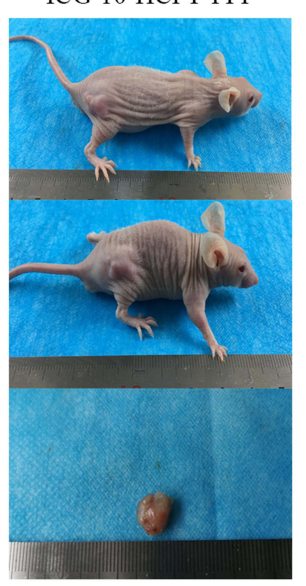

ICG-10-HCPT-PFP+LIFU

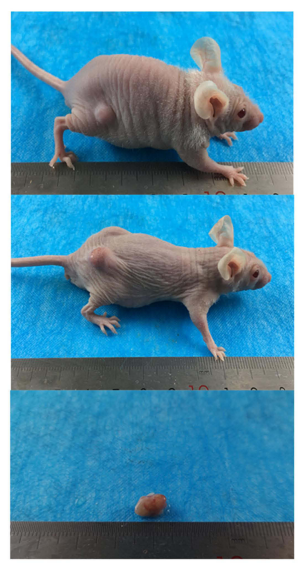

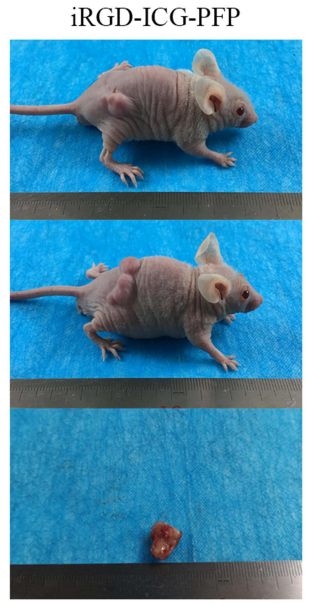

iRGD-ICG-10-HCPT-PFP

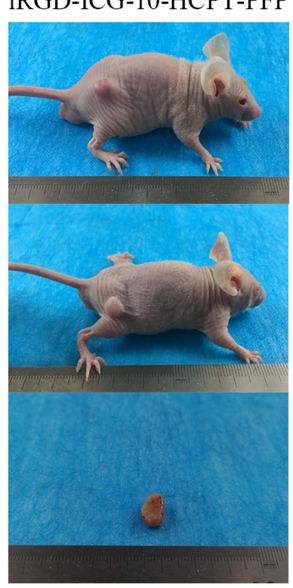

iRGD-ICG-PFP+LIFU

iRGD-ICG-10-HCPT-PFP+LIFU

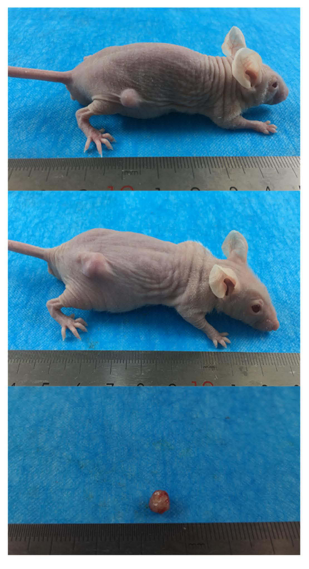

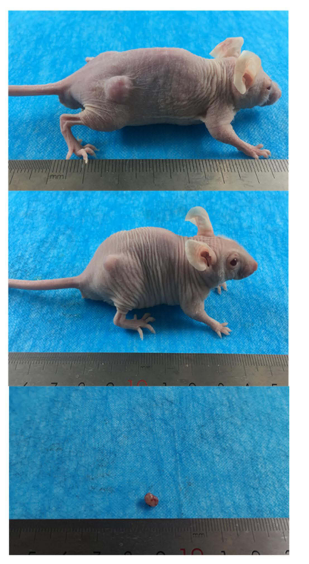

B

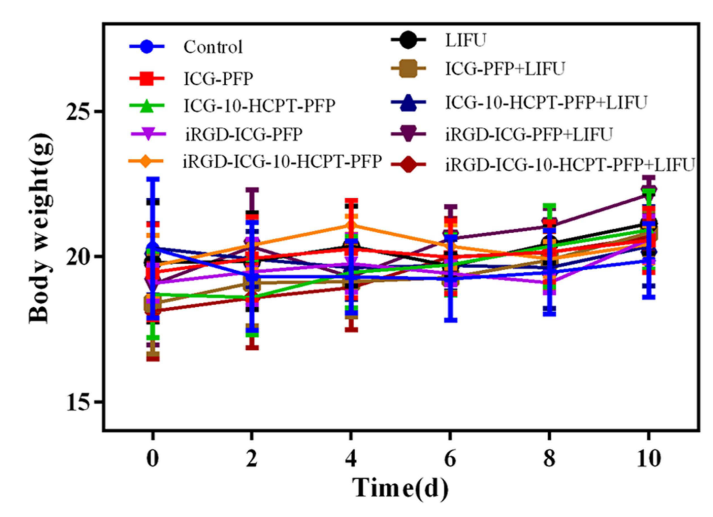

C

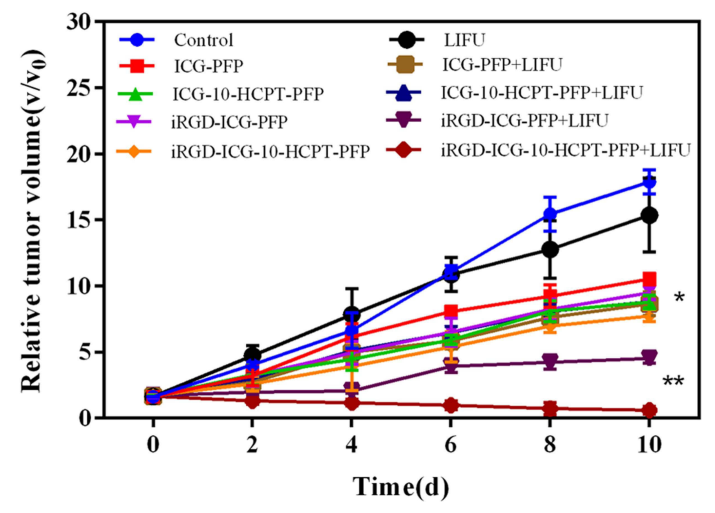

Figure 9 The situation of receiving various nanoparticle treatments of nude mice in each group (A) images of nude mice in each group before and after treatment and pictures of separated tumors after treatment $(\mathbf{B})$ body weight changes of nude mice in each group during treatment $(n=5)$. (C) Changes of tumor volume of nude mice in each group during treatment, $* \mathrm{P}<0.05$ and $* * \mathrm{P}<0.01$.

the second highest, and the difference was significant $(\mathrm{P}<$ 0.01) (Figure $9 \mathrm{~A}$ and $\mathrm{C}$ ). There was no significant difference in tumor size or tumor suppression rate between the control group and the LIFU irradiation-alone group. These results suggest that the iRGD-ICG-10-HCPT-PFP-NPs+ LIFU group had the best therapeutic effect.

In pathological tissue sections (Figure 10A), we found that in H\&E-stained sections of tumors, a large number of 

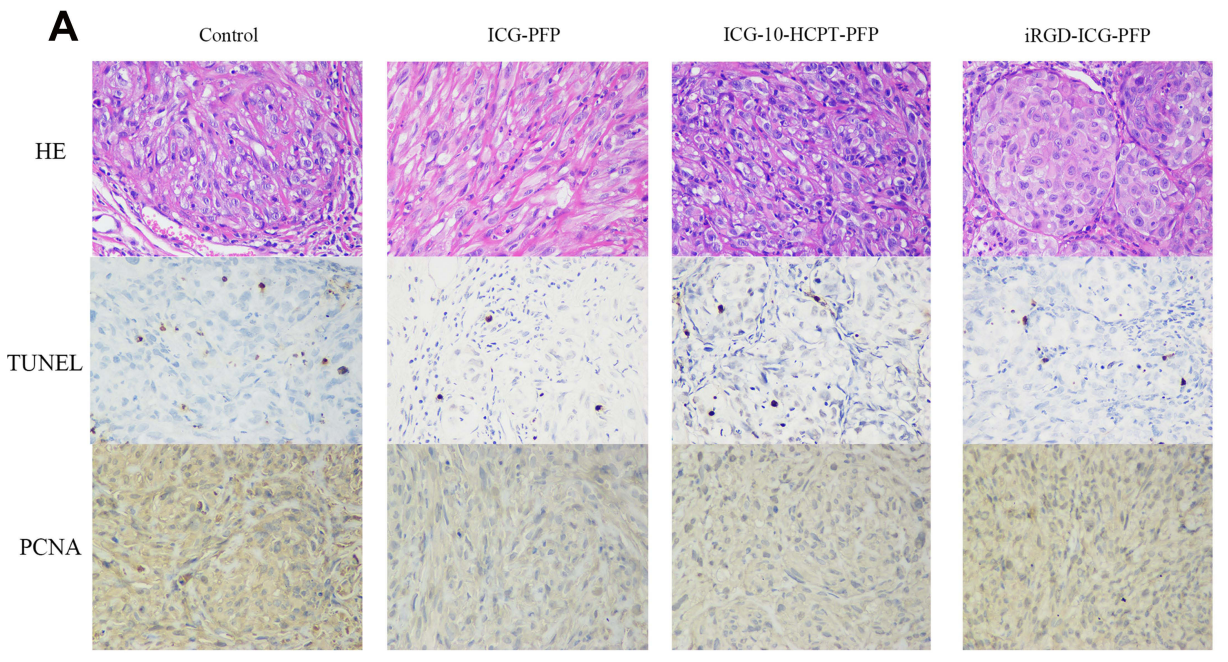

iRGD-ICG-10-HCPT-PFP
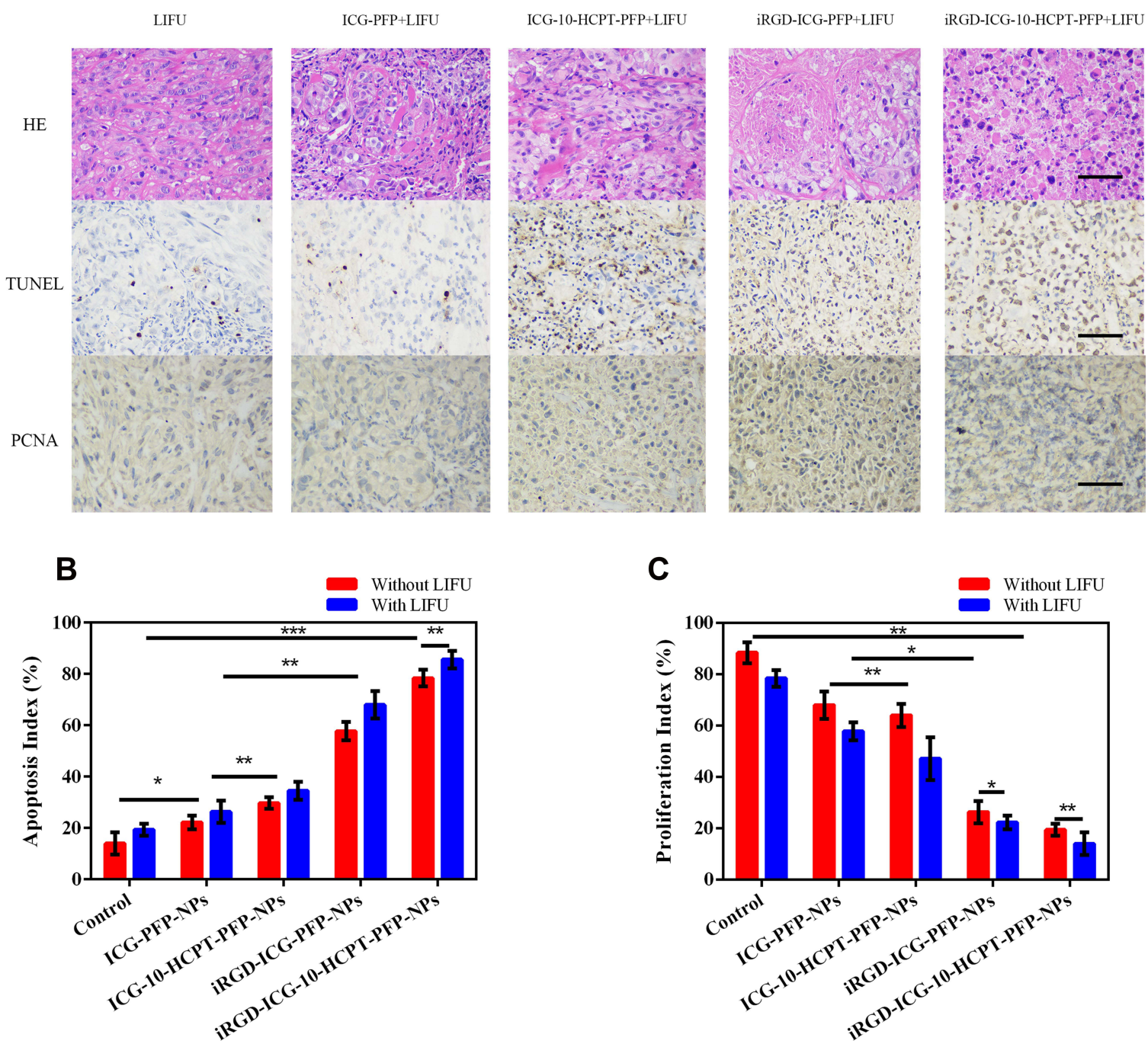

Figure 10 Histopathological section after treatment (A) tumors were isolated after treatment of nude mice in each group, and H\&E staining, TUNEL and PCNA were performed, scale bar: $40 \mu \mathrm{m}$ (B) proapoptotic index statistics (C) antiproliferative index statistics. 
cell ruptures and nuclear fragmentation findings were observed in the iRGD-ICG-10-HCPT-PFP-NPs+LIFU group. In TUNEL staining, the cells were stained brownish-yellow as apoptotic cells, and a large number of brownish-yellow cells were observed in the iRGD-ICG -10-HCPT-PFP-NPs+LIFU group, suggesting the strongest proapoptotic effect on the tumor. In PCNA, the cells were stained brownish-yellow as proliferating cells, and no more significant brownish-yellow cells were seen in the iRGD-ICG-10-HCPT-PFP-NPs+LIFU group, suggesting a significant inhibition of proliferation. The proapoptotic index and antiproliferative index were statistically analyzed separately (Figure $10 \mathrm{~B}$ and C). ${ }^{30}$ Finally, H\&E staining of the viscera of each group showed no significant pathological changes, suggesting that the NPs have good biosafety (Figure 11). By injecting different NPs into tumor-bearing nude mice for treatment, we observed that the body weight of all groups of nude mice did not change significantly, and there was no significant decrease after receiving LIFU irradiation, which indicated the biosafety of NPs and LIFU in vivo. By treatment, we observed that in the iRGD-ICG-10-HCPT-PFP-NPs + LIFU group, the overall tumor inhibition rate was the highest, suggesting that its therapeutic effect was the best, and this result was also consistent with previous in vitro experiments, demonstrating our conjecture about the therapeutic effect of this nanoparticle in vivo. To examine further the therapeutic effect of NPs, the tumors were isolated and pathologically sectioned after sacrificing each group of nude mice. As observed by H\&E staining, there was a large amount of cell fragmentation and karyolysis in the iRGD-ICG-10HCPT-PFP-NPs+LIFU group. Following TUNEL and PCNA staining, the results consistent with in vitro experiments were also obtained, with the iRGD-ICG-10-HCPTPFP-NPs+LIFU group having the best antiproliferative and proapoptotic effects. ${ }^{30,50}$ Therefore, iRGD-ICG-10HCPT-PFP-NPs also had the best therapeutic effect on tumors in vivo after combined LIFU irradiation. After H\&E staining of the organs of nude mice in each group, no obvious pathological changes were observed in each group, indicating that the biosafety of NPs in vivo is also more reliable because all the materials used are biocompatible and easy to metabolize. ${ }^{37}$

\section{Conclusion}

In summary, ultrasonic molecular imaging, as a research hotspot, opens new ideas for the diagnosis and treatment of many diseases and is finding new directions. In some studies of molecular imaging in the past, its direction was mostly single, and some studies were based on therapeutic purposes but were more biased towards pharmaceutical research. ${ }^{38,39}$ In such studies, the prepared nanoscale molecular probes are mainly able to target accurately the disease or tumor site and to release drugs, such as liposomal doxorubicin, which has been put into clinical use. ${ }^{40}$ However, this is only in the field of using nanoscale molecular probes for treatment, and there is a lack of diagnosis for diseases. Therefore, the organic combination of diagnosis and treatment is in line with the direction of future molecular imaging research development. Our research undoubtedly works in this direction.

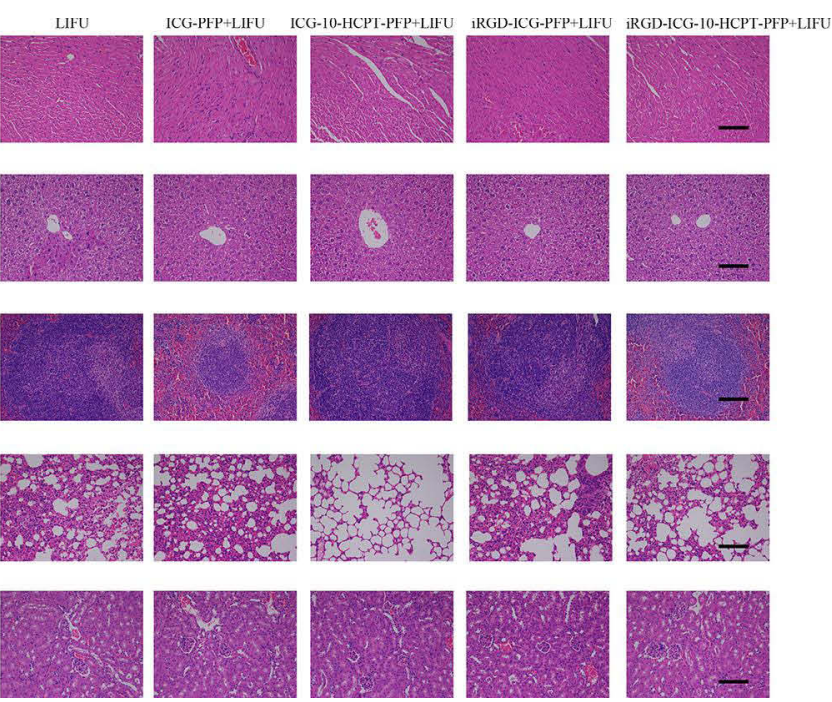

Figure I I Nude mice in each group were sacrificed after nanoparticle injection, the heart, liver, spleen, lung and kidney were isolated, and H\&E sections were performed to observe the safety of nanoparticles; scale bar: $100 \mu \mathrm{m}$. 
The iRGD-ICG-10-HCPT-PFP-NPs are a novel multifunctional molecular probe with regular shape, small particle size, and the ability of ultrasound/PA dual-modality imaging but also to target the tumor area, to penetrate deep into the tumor, and to release the drug under LIFU irradiation, to achieve a better therapeutic effect on the tumor. Our study is based on the results of previous ultrasonic molecular imaging studies, moving towards the goal of integrated diagnosis and treatment and precise treatment and exploring new ideas and methods for the treatment of HCC.

\section{Data Sharing Statement}

All experimental data to support the findings from this study will be made available to interested investigators.

\section{Acknowledgment}

We would like to thank all the members of our laboratory and section for their comments and suggests on this research. We also thank to the Chongqing Key Laboratory of Ultrasound Molecular Imaging at the Second Affiliated Hospital of Chongqing Medical University.

\section{Author Contributions}

Huipu Li: Writing-original draft, Methodology, Conceptualization. Shasha Shi: Formal analysis, Validation, Software. Meng Wu: Validation, Visualization. Wei Shen: Experimental method reference. Jianli Ren: Synthesis method of base material. Zhechuan Mei: Project approval. Haitao Ran: Feasibility assessment. Zhigang Wang: Some project funding. Yi Tian: Review Data. Jian Gao: Resource provision, Data curation. Hongyun Zhao: Writing-original draft, Methodology, Software, Some project funding. All authors made a significant contribution to the work reported, whether that is in the conception, study design, execution, acquisition of data, analysis and interpretation, or in all these areas; took part in drafting, revising or critically reviewing the article; gave final approval of the version to be published; have agreed on the journal to which the article has been submitted; and agree to be accountable for all aspects of the work.

\section{Funding}

Supported by National Natural Science Foundation of China for Youth Scholars. (81801714). Kuanren Talents Program of the Second Affiliated Hospital of Chongqing Medical University (2021240308). Basic research and frontier exploration project of Chongqing science and technology commission (cstc2018jcyjAX0181). Hubei
Provincial Natural Science Foundation (2020CFB729) and Hubei Provincial Health Commission of the Youth Talent Project (WJ2021Q044).

\section{Disclosure}

The authors declare no conflicts of interest in this work. Additionally, the authors declare that they have no known competing financial interests or personal relationships that could have appeared to influence the work reported in this paper.

\section{References}

1. Forner A, Llovet JM, Bruix J. Hepatocellular carcinoma. Lancet. 2012;379(9822):1245-1255. doi:10.1016/S0140-6736(11)61347-0

2. Forner A, Reig M, Bruix J. Hepatocellular carcinoma. Lancet. 2018;391(10127):1301-1314. doi:10.1016/S0140-6736(18)30010-2

3. Llovet JM, Burroughs A, Bruix J. Hepatocellular carcinoma. Lancet. 2003;362(9399):1907-1917. doi:10.1016/S0140-6736(03)14964-1

4. C-y LIU, Chen K-F, Chen P-J. Treatment of liver cancer. Cold Spring Harb Perspect Med. 2015;5(9):a021535. doi:10.1101/cshperspect. a021535

5. Maresca D, Lakshmanan A, Abedi M, et al. Biomolecular ultrasound and sonogenetics. Annu Rev Chem Biomol Eng. 2018;9:229-52.

6. Montesi SB, Désogère P, Fuchs BC, Caravan P. Molecular imaging of fibrosis: recent advances and future directions. $J$ Clin Invest. 2019;129(1):24-33. doi:10.1172/JCI122132

7. Kobayashi D, Hayashida Y, Sano K, et al. Agglomeration and rapid ascent of microbubbles by ultrasonic irradiation. Ultrason Sonochem. 2011;18(5):1193-1196. doi:10.1016/j.ultsonch.2010.11.005

8. Min HS, You DG, Son S, et al. Echogenic glycol chitosan nanoparticles for ultrasound-triggered cancer theranostics. Theranostics. 2015;5(12):1402-1418. doi:10.7150/thno.13099

9. Zylberberg C, Gaskill K, Pasley S, et al. Engineering liposomal nanoparticles for targeted gene therapy. Gene Ther. 2017;24 (8):441-452. doi:10.1038/gt.2017.41

10. D'souza AA, Shegokar R. Polyethylene glycol (PEG): a versatile polymer for pharmaceutical applications. Expert Opin Drug Deliv. 2016;13(9):1257-1275. doi:10.1080/17425247.2016.1182485

11. Kalyane D, Raval N, Maheshwari R, et al. Employment of enhanced permeability and retention effect (EPR): nanoparticle-based precision tools for targeting of therapeutic and diagnostic agent in cancer. Mater Sci Eng C Mater Biol Appl. 2019;98:1252-1276. doi:10.1016/j.msec.2019.01.066

12. Maeda H, Nakamura H, Fang J. The EPR effect for macromolecular drug delivery to solid tumors: improvement of tumor uptake, lowering of systemic toxicity, and distinct tumor imaging in vivo. $A d v$ Drug Deliv Rev. 2013;65(1):71-79. doi:10.1016/j.addr.2012.10.002

13. Danhier F. To exploit the tumor microenvironment: since the EPR effect fails in the clinic, what is the future of nanomedicine? J Control Release. 2016;244(Pt A):108-121. doi:10.1016/j.jconrel.2016.11.015

14. Ruoslahti E. Tumor penetrating peptides for improved drug delivery. Adv Drug Deliv Rev. 2017;110:110-111.

15. Hunt $\mathrm{H}$, Teesalu T. Peritoneal carcinomatosis targeting with tumor homing peptides. Molecules. 2018;23(5):1190.

16. Sugahara KN, Teesalu T, Karmali PP, et al. Tissue-penetrating delivery of compounds and nanoparticles into tumors. Cancer Cell. 2009;16(6):510-520. doi:10.1016/j.ccr.2009.10.013

17. Cho H-J, Park S-J, Y-s LEE, et al. Theranostic iRGD peptide containing cisplatin prodrug: dual-cargo tumor penetration for improved imaging and therapy. $J$ Control Release. 2019;300:73-80. doi:10.1016/j.jconrel.2019.02.043 
18. Lin J, Zhang Y, Wu J, et al. Neuropilin 1 (NRP1) is a novel tumor marker in hepatocellular carcinoma. Clin Chim Acta. 2018;485:158-165. doi:10.1016/j.cca.2018.06.046

19. Jia G, Han Y, An Y, et al. NRP-1 targeted and cargo-loaded exosomes facilitate simultaneous imaging and therapy of glioma in vitro and in vivo. Biomaterials. 2018;178:302-316. doi:10.1016/j.biomaterials.2018. 06.029

20. Lo JH, Hao L, Muzumdar MD, et al. iRGD-guided tumor-penetrating nanocomplexes for therapeutic siRNA delivery to pancreatic cancer. Mol Cancer Ther. 2018;17(11):2377-2388. doi:10.1158/1535-7163. MCT-17-1090

21. Steinberg I, Huland DM, Vermesh O, et al. Photoacoustic clinical imaging. Photoacoustics. 2019;14:77-98. doi:10.1016/j. pacs.2019.05.001

22. Deng L, Cai X, Sheng D, et al. A laser-activated biocompatible theranostic nanoagent for targeted multimodal imaging and photothermal therapy. Theranostics. 2017;7(18):4410-4423. doi:10.7150/ thno. 21283

23. Manohar S, Gambhir SS. Clinical photoacoustic imaging. Photoacoustics. 2020;19:100196. doi:10.1016/j.pacs.2020.100196

24. Kim J, Park E-Y, Park B, et al. Towards clinical photoacoustic and ultrasound imaging: probe improvement and real-time graphical user interface. Exp Biol Med (Maywood). 2020;245(4):321-329. doi:10.1177/1535370219889968

25. Li B, Qin H, Yang S, et al. In vivo fast variable focus photoacoustic microscopy using an electrically tunable lens. Opt Express. 2014;22 (17):20130-20137. doi:10.1364/OE.22.020130

26. Egloff-juras C, Bezdetnaya L, Dolivet G, et al. NIR fluorescence-guided tumor surgery: new strategies for the use of indocyanine green. Int $J$ Nanomedicine. 2019;14:7823-7838. doi:10.2147/IJN.S207486

27. Jin X, Lu X, Zhang Z, et al. Indocyanine green-parthenolide thermosensitive liposome combination treatment for triple-negative breast cancer. Int J Nanomedicine. 2020;15:3193-3206. doi:10.2147/IJN. S245289

28. Li G, Zhao M, Zhao L. Well-defined hydroxyethyl starch-10-hydroxy camptothecin super macromolecule conjugate: cytotoxicity, pharmacodynamics research, tissue distribution test and intravenous injection safety assessment. Drug Deliv. 2016;23(8):2860-2868.

29. Liu Y, Li D, Guo X, et al. A pH-responsive prodrug delivery system of 10-HCPT for controlled release and tumor targeting. Int J Nanomedicine. 2017;12:2227-2242. doi:10.2147/IJN.S125849

30. Zhao $\mathrm{H}$, Wu M, Zhu L, et al. Cell-penetrating peptide-modified targeted drug-loaded phase-transformation lipid nanoparticles combined with low-intensity focused ultrasound for precision theranostics against hepatocellular carcinoma. Theranostics. 2018;8 (7):1892-1910. doi:10.7150/thno.22386

31. Zhang Y, Yong L, Luo Y, et al. Enhancement of HIFU ablation by sonosensitizer-loading liquid fluorocarbon nanoparticles with pre-targeting in a mouse model. Sci Rep. 2019;9(1):6982. doi:10.1038/s41598-019-43416-y

32. Zhu L, Zhao $\mathrm{H}$, Zhou $\mathrm{Z}$, et al. Peptide-functionalized phase-transformation nanoparticles for low intensity focused ultrasound-assisted tumor imaging and therapy. Nano Lett. 2018;18 (3):1831-1841. doi:10.1021/acs.nanolett.7b05087

33. Zhang H. Thin-film hydration followed by extrusion method for liposome preparation. Methods Mol Biol. 2017;1522:17-22.

34. Al-amin MD, Bellato F, Mastrotto F, et al. Dexamethasone loaded liposomes by thin-film hydration and microfluidic procedures: formulation challenges. Int J Mol Sci. 2020;21(5):1611. doi:10.3390/ ijms 21051611
35. Hirschhaeuser F, Menne H, Dittfeld C, et al. Multicellular tumor spheroids: an underestimated tool is catching up again. J Biotechnol. 2010;148(1):3-15. doi:10.1016/j.jbiotec.2010.01.012

36. Ferreira LP, Gaspar VM, Mano JF. Bioinstructive microparticles for self-assembly of mesenchymal stem Cell-3D tumor spheroids. Biomaterials. 2018;185:155-173. doi:10.1016/j. biomaterials.2018.09.007

37. Zhang L, Wang D, Yang K, et al. Mitochondria-targeted artificial "nano-RBCs" for amplified synergistic cancer phototherapy by a single NIR irradiation. Adv Sci (Weinh). 2018;5(8):1800049. doi:10.1002/advs.201800049

38. Zhang J, Hu J, Chan HF, et al. iRGD decorated lipid-polymer hybrid nanoparticles for targeted co-delivery of doxorubicin and sorafenib to enhance anti-hepatocellular carcinoma efficacy. Nanomedicine. 2016;12(5):1303-1311. doi:10.1016/j.nano.2016.01.017

39. Barenholz Y. Doxil ${ }^{\mathbb{R}}$-the first FDA-approved nano-drug: lessons learned. J Control Release. 2012;160(2):117-134. doi:10.1016/j. jconrel.2012.03.020

40. Gabizon AA, Patil Y, La-beck NM. New insights and evolving role of pegylated liposomal doxorubicin in cancer therapy. Drug Resist Updat. 2016;29:90-106. doi:10.1016/j.drup.2016.10.003

41. Negi D, Spiegelberg J, Muto S, et al. Proposal for measuring magnetism with patterned apertures in a transmission electron microscope. Phys Rev Lett. 2019;122(3):37201. doi:10.1103/ PhysRevLett.122.037201

42. Lundquist P, Artursson P. Oral absorption of peptides and nanoparticles across the human intestine: opportunities, limitations and studies in human tissues. Adv Drug Deliv Rev. 2016;106(Pt B):256-276.

43. Jiang T, Zhang Z, Zhang Y, et al. Dual-functional liposomes based on $\mathrm{pH}$-responsive cell-penetrating peptide and hyaluronic acid for tumor-targeted anticancer drug delivery. Biomaterials. 2012;33 (36):9246-9258. doi:10.1016/j.biomaterials.2012.09.027

44. Li J, Xin M, Huo Y, et al. Synthesis of $\beta$-cyclodextrin-PEG-G molecules to delay tumor growth and application of $\beta$-cyclodextrinPEG-G aggregates as drug carrier. Carbohydr Polym. 2020;229:115478. doi:10.1016/j.carbpol.2019.115478

45. Chaudhary Z, Khan GM, Abeer MM, et al. Efficient photoacoustic imaging using indocyanine green (ICG) loaded functionalized mesoporous silica nanoparticles. Biomater Sci. 2019;7(12):5002-5015. doi:10.1039/C9BM00822E

46. Liang X, Fang L, Li X, et al. Activatable near infrared dye conjugated hyaluronic acid based nanoparticles as a targeted theranostic agent for enhanced fluorescence/CT/photoacoustic imaging guided photothermal therapy. Biomaterials. 2017;132:72-84. doi:10.1016/j. biomaterials.2017.04.006

47. Ye S, Wang F, Fan Z, et al. Light/pH-triggered biomimetic red blood cell membranes camouflaged small molecular drug assemblies for imaging-guided combinational chemo-photothermal therapy. ACS Appl Mater Interfaces. 2019;11(17):15262-15275. doi:10.1021/ acsami.9b00897

48. Toccaceli G, Barbagallo G, Peschillo S. Low-intensity focused ultrasound for the treatment of brain diseases: safety and feasibility. Theranostics. 2019;9(2):537-539. doi:10.7150/thno.31765

49. Ding N, Zou Z, Sha H, et al. iRGD synergizes with PD-1 knockout immunotherapy by enhancing lymphocyte infiltration in gastric cancer. Nat Commun. 2019;10(1):1336. doi:10.1038/s41467-01909296-6

50. Rahman M, Al-ghamdi SA, Alharbi KS, et al. Ganoderic acid loaded nano-lipidic carriers improvise treatment of hepatocellular carcinoma. Drug Deliv. 2019;26(1):782-793. doi:10.1080/ 10717544.2019.1606865 


\section{Publish your work in this journal}

The International Journal of Nanomedicine is an international, peerreviewed journal focusing on the application of nanotechnology in diagnostics, therapeutics, and drug delivery systems throughout the biomedical field. This journal is indexed on PubMed Central, MedLine, CAS, SciSearch ${ }^{\mathbb{B}}$, Current Contents ${ }^{\mathbb{B}} /$ Clinical Medicine,
Journal Citation Reports/Science Edition, EMBase, Scopus and the Elsevier Bibliographic databases. The manuscript management system is completely online and includes a very quick and fair peer-review system, which is all easy to use. Visit http://www.dovepress.com/ testimonials.php to read real quotes from published authors.

Submit your manuscript here: https://www.dovepress.com/international-journal-of-nanomedicine-journal 